\title{
COHOMOLOGIE DES FIBRÉS EN DROITES SUR LES COMPACTIFICATIONS DES GROUPES RÉDUCTIFS
}

\author{
PAR AleXis TCHOUDJEM
}

\begin{abstract}
RÉSUMÉ. - On s'intéresse aux compactifications équivariantes d'un groupe réductif quelconque, $G$, complexe et connexe, vu comme espace homogène pour $G \times G$. Pour un revêtement fini $\tilde{G}$ de $G$, les groupes de cohomologie des fibrés en droites sur ces compactifications sont naturellement des $\tilde{G} \times \tilde{G}$ modules de dimension finie. On détermine, dans cet article, tous ces $\tilde{G} \times \tilde{G}$-modules en calculant leur multiplicité selon chaque $\tilde{G} \times \tilde{G}$-module simple. La formule obtenue est en particulier valable pour la compactification magnifique d'un groupe adjoint et généralise aussi la description bien connue de la cohomologie des fibrés en droites sur les variétés toriques complètes. Notre méthode repose sur le complexe de Grothendieck-Cousin, qui, si $\mathfrak{g}$ est l'algèbre de Lie de $G$, est un complexe de $\mathfrak{g} \times \mathfrak{g}$-modules. Pour analyser ces $\mathfrak{g} \times \mathfrak{g}$-modules, on en donne des filtrations dont le gradué associé fait intervenir des «modules de Verma généralisés ».
\end{abstract}

(c) 2004 Elsevier SAS

ABSTRACT. - We consider equivariant compactifications of some reductive complex and connected group, $G$, considered as an homogeneous space for $G \times G$. For a finite covering $\tilde{G}$ of $G$, the cohomology groups of line bundles over those compactifications are naturally finite dimensional $\tilde{G} \times \tilde{G}$-modules. We determine, in this article, all those $\tilde{G} \times \tilde{G}$-modules by calculating their multiplicities along each simple $\tilde{G} \times \tilde{G}$-module. The achieved formula is in particular valid for the wonderful compactification of adjoint groups and also generalizes the well known description of the cohomology of line bundles over complete toric varieties. Our method is based upon the Grothendieck-Cousin complex which, if $g$ is the Lie algebra of $G$, is a $\mathfrak{g} \times \mathfrak{g}$-module complex. We analyse those $\mathfrak{g} \times \mathfrak{g}$-modules by giving some filtrations in the associated graduate of which, some "generalized Verma modules" occur.

(c) 2004 Elsevier SAS

\section{Introduction}

Soit $G$ un groupe réductif et connexe sur $\mathbb{C}$. Le célèbre théorème de Borel-Weil-Bott décrit très simplement la cohomologie des fibrés en droites sur les $G$-variétés de drapeaux, au moyen des représentations irréductibles de $G$. Notre but est d'établir un résultat analogue pour une autre famille de variétés : les compactifications équivariantes du groupe $G$. Comme celui-ci est un espace homogène pour l'action, par multiplication à gauche et à droite, de $G \times G$, ces compactifications admettent une description combinatoire qui résulte de la théorie des plongements des espaces homogènes sphériques (cf. [29]). On peut même obtenir une description combinatoire des fibrés en droites $L$ sur une compactification équivariante $X$. 
Nous exprimons ici les groupes de cohomologie de $L$ sur $X$, en fonction de ces données combinatoires. Pour un revêtement fini $\tilde{G}$ de $G$, tel que $L$ est $\tilde{G} \times \tilde{G}$-linéarisable, ${ }^{1}$ ces groupes $H^{i}(X, L)$ sont en fait des $\tilde{G} \times \tilde{G}$-modules (pour tout $i \geqslant 0$ ). Notre théorème principal (2.1) donne leurs multiplicités selon chaque $\tilde{G} \times \tilde{G}$-module simple. Ce résultat fait intervenir des « objets toriques » et, pour y parvenir, j'utilise le complexe de Grothendieck-Cousin, tel qu'il a été introduit notamment dans [25]. C'est un complexe fait de groupes de cohomologie à support dans les $B \times B^{-}$orbites de $X$ (on note $B$ et $B^{-}$deux sous-groupes de Borel opposés de $G$ ) et dont l'homologie est exactement la cohomologie $H^{*}(X, L)$.

Dans le contexte des variétés de drapeaux, le complexe correspondant permet de retrouver directement le théorème de Borel-Weil-Bott. Pour ce qui concerne les compactifications, on a besoin d'analyser un peu plus les groupes de cohomologie à support qui apparaissent. Si g est l'algèbre de Lie de $G$, ce sont des représentations de $\mathfrak{g} \times \mathfrak{g}$. On en donnera des filtrations avec des gradués associés plus familiers, en se servant de la structure de la compactification. Cela suffira pour calculer les multiplicités cherchées.

Auparavant ( $c f$. la proposition 4.7), ces filtrations auront mis en lumière l'utilité de considérer $G$ dans une compactification, pour préciser la structure de certains $\mathfrak{g} \times \mathfrak{g}$-modules intéressants du point de vue de la théorie des représentations, comme, par exemple, les groupes de cohomologie de $G$ à support dans les doubles classes $B w B^{-}$.

Lorsque $G$ est un tore, les compactifications sont les variétés toriques complètes; dans ce cas, les groupes de cohomologie des fibrés en droites ont été déterminés par Demazure (cf. [14]). Notre résultat est aussi une généralisation de ce théorème de Demazure.

D'autre part, lorsque $G$ est semi-simple adjoint, une compactification particulière de $G$ est sa compactification magnifique construite par De Concini et Procesi. Pour cette compactification, notre résultat a été démontré par la même méthode, de manière indépendante par S. Kato ( $c f$. [24]), et aussi annoncé dans [33].

\section{Notations}

Ici $G$ sera un groupe réductif et connexe sur $\mathbb{C}$, d'algèbre de Lie g. On choisira $B$ un de ses sous-groupes de Borel et $T$ un tore maximal de $B$; on appellera $B^{-}$le sous-groupe de Borel opposé à $B$, relativement à $T$ (i.e. tel que $B^{-} \cap B=T$ ). Soient $\Phi$ et $W$ le système de racines et le groupe de Weyl de $(G, T)$. On notera $\Phi^{+}$l'ensemble des racines positives, $\Phi^{-}$celui des racines négatives, $\rho$ la demi-somme des racines positives, et, si $w \in W, w * \lambda:=w(\lambda+\rho)-\rho$ pour tout caractère $\lambda$ de $T$. Soient $\Delta$ la base de $\Phi$ définie par $B, l$ la fonction longueur correspondante sur $W$ et $w_{0}$ l'élément le plus long de $W$. Le rang de $G$, c'est-à-dire le cardinal de $\Delta$, sera noté $r$.

Les variétés considérées dans ce texte seront des variétés algébriques sur $\mathbb{C}$.

\section{Rappels sur les compactifications de groupes}

Après la définition générale des compatifications de groupes, on s'intéressera surtout aux compactifications régulières. On rappelle cette notion en 1.2 et aussi les données combinatoires qui caractérisent les faisceaux inversibles sur ces variétés.

\subsection{Définition générale}

On appelle compactification (équivariante) de G toute variété complète, normale qui contient $G$ comme ouvert et où l'action par multiplication à gauche et à droite de $G \times G$ sur $G$ se prolonge.

\footnotetext{
${ }^{1}$ Un tel revêtement existe toujours $c f$. [26, remarque p. 67].
} 
Par exemple, si $G=S L(2, \mathbb{C})$ et si $M(2, \mathbb{C})$ est l'espace des matrices d'ordre 2 , la quadrique de $\mathbb{P}^{4}$ :

$$
\mathcal{Q}:=\left\{[M: t] \in \mathbb{P}(M(2, \mathbb{C}) \times \mathbb{C}): \operatorname{det} M=t^{2}\right\}
$$

est munie d'une action de $G \times G$ définie par :

$$
\begin{aligned}
G \times G \times \mathcal{Q} & \longrightarrow \mathcal{Q}, \\
\left(g_{0}, g_{1},[M: t]\right) & \longmapsto\left[g_{0} M g_{1}^{-1}: t\right] .
\end{aligned}
$$

Pour cette action et pour l'inclusion :

$$
\begin{aligned}
G \longrightarrow \mathcal{Q}, \\
M \longmapsto[M: 1],
\end{aligned}
$$

$\mathcal{Q}$ est une compactification lisse de $G$.

Voici un autre exemple : si $G=P G L(2, \mathbb{C})=S L(2, \mathbb{C}) /\{ \pm I d\}$, alors l'espace projectif $\mathbb{P}(M(2, \mathbb{C}))$ est une compactification lisse de $G$.

D'après [3], dans une compactification du groupe $G$, tout point fixé par le tore maximal $T \times T$ appartient à une orbite fermée de $G \times G$. Nous verrons plus loin ( $c f$. la partie 4.3) que c'est un avantage, par rapport aux compactifications d'autres espaces homogènes sphériques, pour déterminer la cohomologie des fibrés en droites.

\subsection{Compactifications régulières}

La notion de variété régulière a été introduite par Bifet, De Concini et Procesi dans [9], $c f$. aussi $[3, \S 1.4]$ et [7] :

DÉfinition 1.- On dit qu'une variété $X$, munie d'une action de $G$ est régulière si les conditions suivantes sont vérifiées :

1. $X$ est lisse et a une $G$-orbite ouverte et dense $X_{G}^{0}$ dont le complémentaire est un diviseur à croisements normaux. On appellera diviseurs limitrophes les composantes irréductibles de $X-X_{G}^{0}$.

2. Chaque adhérence de $G$-orbite est l'intersection transverse des diviseurs limitrophes qui la contiennent.

3. Si $x \in X$, alors sur l'espace normal à $G \cdot x$ dans $X$, le groupe d'isotropie de $x$ agit avec une orbite dense.

DÉFINITION 2. - Les compactifications régulières de $G$ sont les compactifications (équivariantes) $X$ de $G$ qui sont régulières comme $G \times G$-variétés.

Exemples. -

- Les variétés toriques complètes lisses sont les compactifications régulières des tores.

- La compactification $\mathcal{Q}$ de 1.1 est une compactification régulière.

- Pour un groupe adjoint $G$ (i.e. de centre trivial), De Concini et Procesi ont construit ( $c f$. [13]) une compactification lisse, « canonique », ou « magnifique », $\bar{G}$, de $G$; on peut la définir comme l'unique compactification régulière de $G$ avec une seule $G \times G$-orbite fermée. L'espace projectif $\mathbb{P}(M(2, \mathbb{C}))$ est, par exemple, la compactification magnifique de $P G L(2, \mathbb{C})$.

Jusquà la fin $\mathbf{X}$ sera une compactification régulière de $G$.

Avant d'énoncer le théorème principal sur les groupes de cohomologie, fixons les notations qui concernent $\mathbf{X}$ : 


\subsubsection{Caractérisation combinatoire de la compactification}

On fait d'abord appel à deux réseaux en dualité : le réseau $\mathcal{X}$ des caractères de $T$ et celui, $\mathcal{Y}$, des sous-groupes à un paramètre de $T$. On posera $\mathcal{X}_{\mathbb{R}}:=\mathcal{X} \underset{\mathbb{Z}}{\otimes} \mathbb{R}$ et $\mathcal{Y}_{\mathbb{R}}:=\mathcal{Y} \underset{\mathbb{Z}}{\otimes} \mathbb{R}$. On notera :

$$
\langle,\rangle: \mathcal{X}_{\mathbb{R}} \times \mathcal{Y}_{\mathbb{R}} \rightarrow \mathbb{R}
$$

le crochet de dualité usuel.

Soit :

$$
\mathcal{C}^{+}:=\left\{\nu \in \mathcal{Y}_{\mathbb{R}}: \forall \alpha \in \Delta,\langle\alpha, \nu\rangle \geqslant 0\right\}
$$

l'adhérence de la chambre de Weyl positive.

On va définir une subdivision de $\mathcal{C}^{+}$associée à $\mathbf{X}$.

Pour cela, soit $\bar{T}$ l'adhérence de $T$ dans $\mathbf{X}$. Sur $G$, la restriction de l'action de $G \times G$ à la diagonale de $T, \operatorname{diag}(T)$, est donnée par :

$$
\forall g \in G, \forall t \in T, \quad(t, t) . g=t g t^{-1} .
$$

Elle se prolonge à $\mathbf{X}$. La variété des points fixés par le tore diagonal $\operatorname{diag}(T)$ est lisse $(c f$. [22, prop. 1.3]) et $\bar{T}$ en est une composante irréductible. Pour l'action de $T$ à gauche (i.e. pour l'action de $T \times\{1\}), \bar{T}$ est donc une variété torique complète lisse; on note $\mathcal{E}$ l'éventail associé à $\bar{T}$. Comme $\bar{T}$ est complète, $\mathcal{E}$ est une subdivision de $\mathcal{Y}_{\mathbb{R}}$ (i.e. : $\bigcup_{\sigma \in \mathcal{E}} \sigma=\mathcal{Y}_{\mathbb{R}}$ ) et comme $\bar{T}$ est invariant par l'action de la diagonale du groupe de Weyl :

$$
\operatorname{diag}(W):=\{(w, w): w \in W\}
$$

$\mathcal{E}$ est aussi $W$-invariant. Il résulte de [3, prop. A2] que $\mathcal{E}=W \mathcal{E}^{+}$où $\mathcal{E}^{+}$est la subdivision de $\mathcal{C}^{+}$formée des cônes de $\mathcal{E}$ contenus dans $\mathcal{C}^{+}$. De manière analogue au cas torique, les cônes de l'éventail $\mathcal{E}^{+}$paramètrent des orbites : les $G \times G$-orbites de $\mathbf{X}$ ( $c f$. encore [3, prop. A2]). Pour tout $\sigma \in \mathcal{E}^{+}$, on désigne par $z_{\sigma}$ le point-base correspondant dans $\bar{T}$ et $\operatorname{par} \mathcal{O}_{\sigma}$ sa $G \times G$-orbite. On dira que $z_{\sigma}$ est le point-base de l'orbite $\mathcal{O}_{\sigma}$. Parfois on notera $z_{\mathcal{O}_{\sigma}}$ le point $z_{\sigma}$.

Les $G \times G$-orbites fermées de $\mathbf{X}$ correspondent aux cônes de $\mathcal{E}^{+}$de dimension maximale $r$. Lorsque $\sigma$ est un tel cône, $z_{\sigma}$ a pour groupe d'isotropie $B^{-} \times B$; d'où :

$$
(G \times G) \cdot z_{\sigma} \simeq G / B^{-} \times G / B
$$

(cf. [3, prop. A1 ii)]).

Remarque. - Réciproquement, toute subdivision de $\mathcal{C}^{+}$dont tous les cônes sont lisses (c'est-àdire engendrés par un début de base du réseau $\mathcal{Y}$ ) correspond à une compactification régulière de $G$. Par exemple, la subdivision triviale formée de $\mathcal{C}^{+}$et de ses faces définit la compactification magnifique de $G$ (si $G$ est adjoint).

On va maintenant préciser les « objets toriques » correspondant aux faisceaux inversibles sur X.

\subsubsection{Caractérisation combinatoire des faisceaux inversibles}

On choisit au préalable une isogénie $r: \tilde{G} \rightarrow G$ telle que $\operatorname{Pic}(\tilde{G})=\{0\}$ (cf. [23] ou [26, prop. 4.6]). Tous les faisceaux inversibles sur $\mathbf{X}$ sont alors $\tilde{G} \times \tilde{G}$-linéarisables.

On notera $\tilde{B}, \tilde{B^{-}}, \tilde{T}, \tilde{\mathcal{X}}, \tilde{\mathcal{Y}}$ les ensembles correspondants à $B, B^{-}, T, \mathcal{X}, \mathcal{Y}$ pour $\tilde{G}$. 
Grâce à la paramétrisation des fibrés en droites sur les variétés sphériques de [1, Section 2.2], on obtient ${ }^{2}$ celle des fibrés en droites sur $\mathbf{X}$ par les fonctions linéaires par morceaux sur $\mathcal{C}^{+}$, adaptées à la subdivision $\mathcal{E}^{+}$. Il s'agit des applications $h: \mathcal{C}^{+} \rightarrow \mathbb{R}$ telles que pour tout cône $\sigma$ de $\mathcal{E}^{+}$et tout $n \in \sigma, h(n)=\left\langle h_{\sigma}, n\right\rangle$, pour un certain $h_{\sigma} \in \mathcal{X}_{\mathbb{R}}$.

THÉORÈME $1.1[1, \S 2.2]$. - Étant donnée une fonction linéaire par morceaux $h: \mathcal{C}^{+} \rightarrow \mathbb{R}$ à valeurs entières sur $\tilde{\mathcal{Y}} \cap \mathcal{C}^{+}$et adaptée à l'éventail $\mathcal{E}^{+}$, il existe un faisceau $\mathcal{L}$, inversible et $\tilde{G} \times \tilde{G}$-linéarisé sur $\mathbf{X}$, tel que, pour toute orbite fermée $\mathcal{O}_{\sigma}\left(\sigma\right.$ cône maximal de $\left.\mathcal{E}^{+}\right)$, le groupe $\widehat{B^{-}} \times \tilde{B}$ opère avec le caractère $\left(h_{\sigma},-h_{\sigma}\right)$ dans la fibre $\left.\mathcal{L}\right|_{z_{\sigma}}$.

$\grave{A}$ isomorphisme de faisceaux $\tilde{G} \times \tilde{G}$-linéarisés sur $\mathbf{X}$ près, $\mathcal{L}$ est unique et on le note $: \mathcal{L}_{h}$. On obtient ainsi, à isomorphisme de $\mathfrak{D}_{\mathbf{X}}$-modules près, tous les faisceaux inversibles sur $\mathbf{X}$.

On conservera cette notation $\mathcal{L}_{h}$ pour le faisceau inversible et $\tilde{G} \times \tilde{G}$-linéarisé sur $\mathbf{X}$ correspondant à la fonction linéaire par morceaux $h$.

Remarque. - D'après [27, lemme 2.2], deux linéarisations (pour $\tilde{G} \times \tilde{G}$ ) d'un même faisceau inversible $\mathcal{L}$ sur $\mathbf{X}$ diffèrent d'un caractère de $\tilde{G} \times \tilde{G}$. En conséquence, c'est à translation près par un caractère de $\tilde{G}$ que le faisceau $\mathcal{L}$ définit la fonction $h$.

\section{Description de la cohomologie des faisceaux inversibles sur une compactification de groupe}

En premier lieu, on énonce le théorème principal pour une compactification régulière. Ensuite, on en donnera une version dans le cas particulier des compactifications magnifiques.

Remarque.-Si $X$ est une compactification équivariante quelconque de $G$ (i.e. seulement normale), alors, d'après [2], il existe une compactification régulière $\tilde{\mathbf{X}}$ de $G$ et un morphisme propre et birationnel $\pi: \tilde{\mathbf{X}} \rightarrow X$ tel que :

$$
\text { 1) } \pi_{*} \mathfrak{D}_{\tilde{\mathbf{x}}}=\mathfrak{D}_{X}, \quad \text { 2) } \quad \forall i>0, \quad R^{i} \pi_{*} \mathfrak{D}_{\tilde{\mathbf{x}}}=0 .
$$

En particulier, pour tout faisceau inversible $\mathcal{L}$ sur $X$, on a : $H^{i}(X, \mathcal{L})=H^{i}\left(\tilde{\mathbf{X}}, \pi^{*} \mathcal{L}\right)$ pour tout $i$. Notre théorème 2.1 est donc valable, en toute généralité, pour les groupes de cohomologie des fibrés en droites sur les compactifications équivariantes de $G$.

\subsection{Cas régulier}

Soit $h$ une fonction linéaire par morceaux $\operatorname{sur} \mathcal{C}^{+}$, adaptée à $\mathcal{E}^{+}$et à valeurs entières sur $\tilde{\mathcal{Y}} \cap \mathcal{C}^{+}$.

Les groupes de cohomologie $H^{i}\left(\mathbf{X}, \mathcal{L}_{h}\right)$ sont des modules de dimension finie sous le groupe $\tilde{G} \times \tilde{G}$. Ils se décomposent donc en somme directe de représentations irréductibles. Notons $\mathcal{X}^{+}$ (respectivement $\tilde{\mathcal{X}}^{+}$) l'ensemble des caractères dominants de $\mathcal{X}$ (respectivement de $\tilde{\mathcal{X}}$ ). Pour chaque $\mu \in \tilde{\mathcal{X}}^{+}$, soit $L(\mu)$ le $\tilde{G}$-module simple de plus haut poids $\mu$. ${ }^{3}$ Les seuls modules simples apparaissant dans la décomposition des groupes de cohomologie $H^{i}\left(\mathbf{X}, \mathcal{L}_{h}\right)$ sont les

$$
\operatorname{End}(L(\mu))=L(\mu) \otimes L\left(-w_{0} \mu\right), \quad \mu \in \tilde{\mathcal{X}}^{+} \text {. }
$$

\footnotetext{
${ }^{2}$ On utilise en particulier que $\mathbf{X}$ admet un recouvrement par des ouverts isomorphes à des espaces affines et les trivialisations des fibrés en droites restreints à ces ouverts.

${ }^{3}$ Plus généralement pour chaque $\mu \in \mathcal{X}^{+}$, soit $L(\mu)$ le $\mathfrak{g}$-module simple de plus haut poids $\mu$.
} 
Nous allons exprimer leurs multiplicités au moyen des ensembles :

$$
V(h, \lambda):=\left\{n \in \mathcal{C}^{+}:\langle\lambda, n\rangle-h(n)>0\right\}
$$

où $\lambda \in \mathcal{X}_{\mathbb{R}}$. Pour abréger, on note $h+\mathcal{X}$ l'ensemble des caractères $\mu \in \mathcal{X}_{\mathbb{R}}$ tels que $\mu-h_{\sigma} \in \mathcal{X}$ pour tout cône maximal $\sigma$ de $\mathcal{E}^{+}$. On pose aussi

$$
J_{t}:=\left\{\alpha \in \Delta: t^{-1}(\alpha)<0\right\}
$$

et, pour chaque $\alpha \in \Delta$,

$$
\alpha^{\perp}:=\left\{n \in \mathcal{C}^{+}:\langle\alpha, n\rangle=0\right\} .
$$

On utilisera la notation $H^{*}($,$) pour les groupes de cohomologie relative de parties de \mathcal{Y}_{\mathbb{R}}$ à valeurs dans $\mathbb{R}$ ( $c f$. par exemple [32, cha. 5, sec. 4, ex. 5]).

Cela étant posé :

THÉORÈME 2.1. - On a un isomorphisme de $\tilde{G} \times \tilde{G}$-modules :

$$
H^{i}\left(\mathbf{X}, \mathcal{L}_{h}\right) \simeq \bigoplus_{\mu \in \tilde{\mathcal{X}}^{+}} m_{h}^{i}(\mu) \operatorname{End}(L(\mu))
$$

où pour tout poids entier dominant $\mu$ et tout entier $i$, la multiplicité $m_{h}^{i}(\mu)$ est égale à :

$$
\sum_{t \in W \backslash\{1\}} \operatorname{dim} H^{i-2 l(t)-1}\left(V(h, t * \mu), V(h, t * \mu) \cap \bigcup_{\alpha \in J_{t}} \alpha^{\perp}\right)+\operatorname{dim} H^{i}\left(\mathcal{C}^{+}, V(h, \mu)\right)
$$

si $\mu \in h+\mathcal{X}$ et $m_{h}^{i}(\mu)=0$ sinon.

Afin d'illustrer cette formule compliquée, voici quelques remarques et exemples :

1. Avec un $\tilde{G}$ choisi de sorte que pour tout $\mu \in \tilde{X}$ et pour toute coracine $\alpha^{\vee}$, on ait : $\left\langle\mu, \alpha^{\vee}\right\rangle \in \mathbb{Z}$, la condition $\mu \in h+\mathcal{X}$ s'écrit :

$$
\forall n \in \mathcal{Y} \cap \mathcal{C}^{+}, \quad\langle\mu, n\rangle-h(n) \in \mathbb{Z} .
$$

2. Lorsque $G=T$, on a dans ce cas :

$$
W=\{1\}, \quad \Delta=\emptyset \quad \text { et } \quad \mathcal{C}^{+}=\mathcal{Y}_{\mathbb{R}} .
$$

On retrouve le résultat de Demazure (cf. par exemple [30, th. 2.6]) :

$$
m_{h}^{i}(\mu)=\operatorname{dim} H^{i}\left(\mathcal{Y}_{\mathbb{R}}, V(h, \mu)\right) .
$$

Toutefois, ce résultat est en fait utilisé dans la démonstration ( $c f$. la section 5.4).

3. Lorsque $i=0$, on obtient tout de suite :

$$
m_{h}^{0}(\mu)= \begin{cases}1 & \text { si pour tout } n \in \mathcal{C}^{+},\langle\mu, n\rangle \leqslant h(n) \text { et si } \mu \in h+\mathcal{X} \\ 0 & \text { sinon. }\end{cases}
$$

C’est un résultat déjà connu ( $c f$. par exemple [8, th. du §3.4]). 


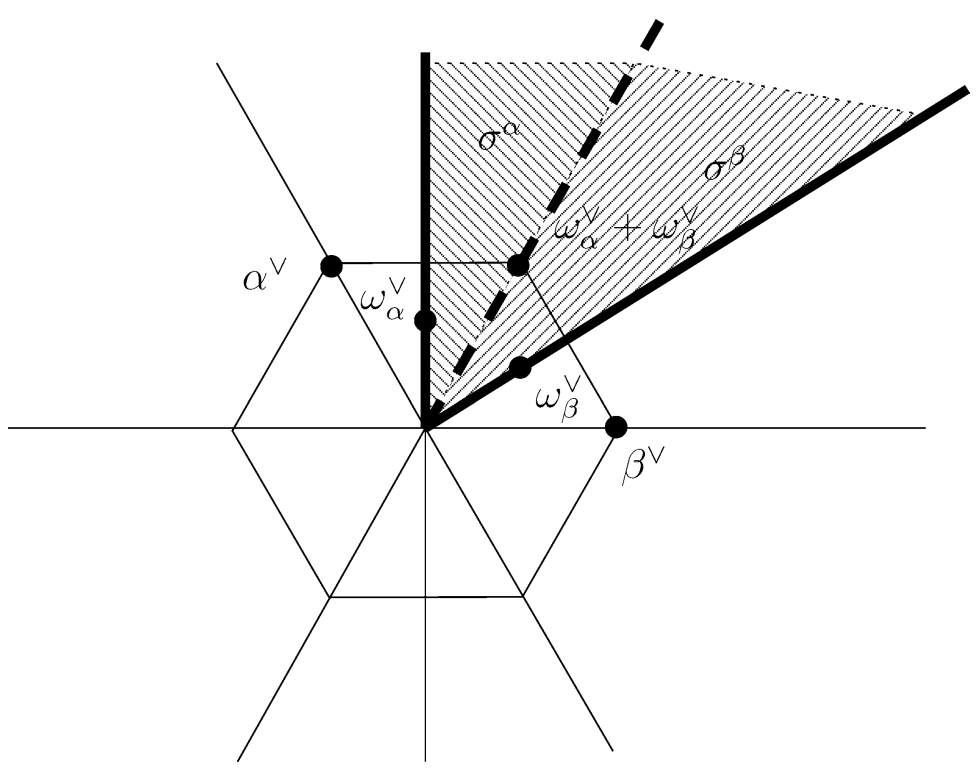

Fig. 1. L'éventail $\mathcal{E}^{+}$.

4. Soit $X\left(\mathcal{E}^{+}\right)$la variété torique associée à l'éventail $\mathcal{E}^{+}$. C'est un ouvert de $\bar{T}$ et, lorsque $i=0,1$, ou 2 , on obtient :

$$
m_{h}^{i}(\mu)=\operatorname{dim}\left(H^{i}\left(X\left(\mathcal{E}^{+}\right), \mathcal{L}_{h}\right)\right)_{\mu}
$$

la dimension de l'espace propre associé au caractère $\mu$.

5. On va maintenant voir un exemple de calcul où l'on trouve une multiplicité $>1$. Lorsque $G=P G L(3, \mathbb{C})$, et $\tilde{G}=S L(3, \mathbb{C})$, les groupes de cohomologie des fibrés en droites sur la compactification magnifique $\bar{G}$ sont des représentations de $S L(3, \mathbb{C}) \times S L(3, \mathbb{C})$ sans multiplicité (c'est-à-dire que leurs multiplicités sont 0 ou 1), $c f$. la remarque 4 . Ce n'est plus le cas pour les compactifications générales du même groupe.

Soient $\alpha, \beta$ les éléments de la base $\Delta$. On note $\left\{\omega_{\alpha}^{\vee}, \omega_{\beta}^{\vee}\right\}$ la base duale de $\{\alpha, \beta\}$ dans $\mathcal{Y}$. Soit X l'éclaté de la compactification magnifique de $G$ le long de son unique $G \times G$-orbite fermée. C'est aussi la compactification régulière de $G$ associée à la subdivision suivante de la chambre de Weyl positive :

$$
\mathcal{C}^{+}=\sigma^{\alpha} \bigcup \sigma^{\beta}
$$

où :

$$
\sigma^{\alpha}:=\mathbb{R}_{+} \omega_{\alpha}^{\vee}+\mathbb{R}_{+}\left(\omega_{\alpha}^{\vee}+\omega_{\beta}^{\vee}\right)
$$

et :

$$
\sigma^{\beta}:=\mathbb{R}_{+}\left(\omega_{\alpha}^{\vee}+\omega_{\beta}^{\vee}\right)+\mathbb{R}_{+} \omega_{\beta}^{\vee} .
$$

Soit $h$ la fonction définie par :

$$
h(n)= \begin{cases}\langle\beta-2 \alpha, n\rangle & \text { si } n \in \sigma^{\alpha}, \\ \langle\alpha-2 \beta, n\rangle & \text { si } n \in \sigma^{\beta}\end{cases}
$$


(il n'y a pas d'ambiguïté). Notons $\mathcal{L}_{h}$ le faisceau inversible correspondant sur $\mathbf{X}$.

On va montrer que la multiplicité de la représentation triviale $\operatorname{End}(L(0))$ dans $H^{3}\left(\mathbf{X}, \mathcal{L}_{h}\right)$ vaut 2 .

On remarque d'abord que le caractère $0 \in h+\mathcal{X}$. Ensuite, puisque si $t \in W$ :

$$
3-2 l(t)-1 \geqslant 0 \quad \Leftrightarrow \quad l(t) \leqslant 1,
$$

on a d'après le même théorème :

$$
\begin{aligned}
m_{h}^{3}(0)= & \sum_{t \in\left\{s_{\alpha}, s_{\beta}\right\}} \operatorname{dim} H^{0}\left(V(h, t * 0), V(h, t * 0) \cap \bigcup_{\delta \in J_{t}} \delta^{\perp}\right) \\
& +\operatorname{dim} H^{3}\left(\mathcal{C}^{+}, V(h, 0)\right) .
\end{aligned}
$$

Or, d'une part :

$$
\begin{aligned}
V(h, 0) & =\left\{n \in \mathcal{C}^{+}: h(n)<0\right\} \\
& =\left\{n_{\alpha} \omega_{\alpha}^{\vee}+n_{\beta} \omega_{\beta}^{\vee}:\left(n_{\alpha}, n_{\beta}\right) \in \mathbb{R}_{+}^{2} \backslash\{(0,0)\}\right\}
\end{aligned}
$$

donc : $H^{3}\left(\mathcal{C}^{+}, V(h, 0)\right)=\{0\}$.

D'autre part, on montre que :

$$
\begin{aligned}
V\left(h, s_{\alpha} * 0\right) & =\left\{n \in \mathcal{C}^{+}:-\langle\alpha, n\rangle-h(n)>0\right\} \\
& =\left\{n_{\alpha} \omega_{\alpha}^{\vee}+n_{\beta} \omega_{\beta}^{\vee}:\left(n_{\alpha}, n_{\beta}\right) \in \mathbb{R}_{+}^{2} \backslash\{(x, x): x \geqslant 0\}\right\}
\end{aligned}
$$

et :

$$
V\left(h, s_{\alpha} * 0\right) \cap \alpha^{\perp}=\mathbb{R}_{+}^{*} \omega_{\beta} .
$$

D'où :

$$
\begin{aligned}
& H^{0}\left(V\left(h, s_{\alpha} * 0\right), V\left(h, s_{\alpha} * 0\right) \cap \alpha^{\perp}\right) \\
& \quad=H^{0}\left(\mathbb{R}_{+} \times \mathbb{R}_{+}^{*},\{0\} \times \mathbb{R}_{+}^{*}\right) \oplus H^{0}\left(\mathbb{R}_{+}^{*} \times \mathbb{R}_{+}, \emptyset\right)=\mathbb{R} .
\end{aligned}
$$

On montre de même, que :

$$
V\left(h, s_{\beta} * 0\right)=\left\{n_{\alpha} \omega_{\alpha}^{\vee}+n_{\beta} \omega_{\beta}^{\vee}:\left(n_{\alpha}, n_{\beta}\right) \in \mathbb{R}_{+}^{2} \backslash\{(x, x): x \geqslant 0\}\right\}
$$

et que :

$$
V\left(h, s_{\beta} * 0\right) \cap \beta^{\perp}=\mathbb{R}_{+}^{*} \omega_{\alpha} .
$$

Donc: $H^{0}\left(V\left(h, s_{\beta} * 0\right), V\left(h, s_{\beta} * 0\right) \cap \beta^{\perp}\right)=\mathbb{R}$.

On a ainsi : $m_{h}^{3}(0)=2$.

\subsection{Cas de la compactification magnifique}

Soit $G_{a d}$ le groupe adjoint de $G$ (le quotient de $G$ par son centre) et $\overline{G_{a d}}$ sa compactification magnifique ( $c f$. la section 1.2). On peut prendre pour $\widetilde{G_{a d}}$ le revêtement universel de $G_{a d}$. Les faisceaux inversibles sur $\overline{G_{a d}}$ sont alors simplement donnés par un poids entier $\lambda \in \tilde{\mathcal{X}}$. Soit $z$ l'unique point fixe de $\bar{G}_{a d}$ pour $B^{-} \times B$ et pour chaque $\lambda \in \tilde{\mathcal{X}}$, soit $\mathcal{L}_{\lambda}$ le faisceau inversible et $\widetilde{G_{a d}} \times \widetilde{G_{a d}}$-linéarisé sur $\overline{G_{a d}}$ tel que le groupe $\widetilde{B^{-}} \times \tilde{B}$ opère dans la fibre $\left.\mathcal{L}\right|_{z}$ avec le caractère $(\lambda,-\lambda)$. 
On note aussi $Q:=\sum_{\alpha \in \Delta} \mathbb{Z} \alpha$ le réseau radiciel et pour chaque $t \in W$,

$$
Q_{t}:=\left\{\sum_{\alpha \in \Delta} \gamma_{\alpha} \alpha \in Q: \gamma_{\alpha}>0 \Leftrightarrow t^{-1}(\alpha)<0\right\} .
$$

On a :

THÉORÈME 2.2. - Comme $\widetilde{G_{a d}} \times \widetilde{G_{a d}}$-modules, pour tout $i \geqslant 0$ :

$$
H^{i}\left(\overline{G_{a d}}, \mathcal{L}_{\lambda}\right)=\bigoplus_{\mu \in \tilde{\mathcal{X}}^{+}} m_{\lambda}^{i}(\mu) \operatorname{End}(L(\mu))
$$

avec chaque multiplicité $m_{\lambda}^{i}(\mu)$ valant le nombre de $t \in W$ tels qu'à la fois $2 l(t)+\left|J_{t}\right|=i$ et $t * \mu \in \lambda+Q_{t}$.

Ce théorème 2.2 est un cas particulier du théorème 2.1 comme nous allons le voir tout de suite. On remarque d'abord que, dans ce cas, $h$ est simplement un caractère $\lambda$ de $\tilde{\mathcal{X}}$. Soit $\mu \in \tilde{\mathcal{X}}^{+}$. On a alors :

$$
V(\lambda, \mu)=\left\{n \in \mathcal{C}^{+}:\langle\mu, n\rangle-\langle\lambda, n\rangle>0\right\} .
$$

La condition $\mu \in h+\mathcal{X}$ du théorème signifie que $\mu-\lambda \in \mathcal{X}$.

On fixe ensuite $\nu \in \mathcal{X}$. Soit $V:=\left\{n \in \mathcal{C}^{+}:\langle\nu, n\rangle>0\right\}$. On va montrer (et cela suffira) que, pour tout $j \geqslant 0$ et tout $t \in W \backslash\{1\}$ :

$$
H^{j}\left(V, V \cap \bigcup_{\alpha \in J_{t}} \alpha^{\perp}\right)=\mathbb{R} \quad \text { si } j=\left|J_{t}\right|-1 \text { et } \operatorname{si} \nu \in Q_{t}
$$

et que $H^{j}\left(V, V \cap \bigcup_{\alpha \in J_{t}} \alpha^{\perp}\right)=\{0\}$ dans tous les autres cas.

Comme cela est vrai lorsque $V=\emptyset$, on va maintenant supposer que $V$ n'est pas vide.

Puisque $V$ est convexe, on a, pour tout $j$ :

$$
H^{j}\left(V, V \cap \bigcup_{\alpha \in J_{t}} \alpha^{\perp}\right)=\tilde{H}^{j-1}\left(V \cap \bigcup_{\alpha \in J_{t}} \alpha^{\perp}\right)
$$

où $\tilde{H}^{*}()$ désigne la cohomologie réduite ( $c f$. par exemple [32, chap. 5 , sec. $\left.\left.4, \S 2\right]\right)$.

Par l'intermédiaire de la base duale $\left\{\omega_{\alpha}^{\vee}: \alpha \in \Delta\right\}$ de $\Delta$ dans $\mathcal{Y}$, on identifie $\mathcal{C}^{+}$avec $\mathbb{R}_{+}^{\Delta}$ et on définit une fonction :

$$
p:\left\{\begin{array}{l}
\mathcal{C}^{+} \longrightarrow \bigoplus_{\delta \in J_{t}} \mathbb{R}_{+} \omega_{\delta}^{\vee} \simeq \mathbb{R}_{+}^{J_{t}}, \\
n=\sum_{\delta \in \Delta} n_{\delta} \omega_{\delta}^{\vee} \longmapsto \sum_{\delta \in J_{t}} n_{\delta} \omega_{\delta}^{\vee} .
\end{array}\right.
$$

Cette application $p$ est continue et on s'aperçoit que, restreinte à $V \cap \bigcup_{\alpha \in J_{t}} \alpha^{\perp}$, ses fibres sont convexes :

$$
\begin{gathered}
\forall n^{0} \in \mathbb{R}_{+}^{J_{t}}, \quad\left\{n \in V \cap \bigcup_{\alpha \in J_{t}} \alpha^{\perp}: p(n)=n^{0}\right\}=\emptyset \\
\text { ou } \quad\left\{n=n^{1}+n^{0}: n^{1} \in \mathbb{R}_{+}^{\Delta \backslash J_{t}} \text { et }\left\langle\nu, n^{1}\right\rangle>-\left\langle\nu, n^{0}\right\rangle\right\}
\end{gathered}
$$


(on a identifié $\bigoplus_{\alpha \in \Delta \backslash J_{t}} \mathbb{R}_{+} \omega_{\delta}^{\vee}$ avec $\mathbb{R}_{+}^{\Delta \backslash J_{t}}$ ). On en déduit grâce à une suite spectrale de Leray (cf. [19, chap. II, théorème 4.17.1]) que :

$$
\tilde{H}^{j-1}\left(V \cap \bigcup_{\alpha \in J_{t}} \alpha^{\perp}\right)=\tilde{H}^{j-1}\left(p\left(V \cap \bigcup_{\alpha \in J_{t}} \alpha^{\perp}\right)\right)
$$

pour tout $j$.

Mais, si on note $\partial \mathbb{R}_{+}^{J_{t}}$ le bord de $\mathbb{R}_{+}^{J_{t}}$, les égalités suivantes sont vérifiées :

$$
\begin{aligned}
p\left(V \cap \bigcup_{\alpha \in J_{t}} \alpha^{\perp}\right) & =\left\{n \in \mathbb{R}_{+}^{J_{t}} \cap \bigcup_{\alpha \in J_{t}} \alpha^{\perp}: \exists n^{1} \in \mathbb{R}_{+}^{\Delta \backslash J_{t}},\langle\nu, n\rangle+\left\langle\nu, n^{1}\right\rangle>0\right\} \\
& =\mathbb{R}_{+}^{J_{t}} \cap\left(\bigcup_{\alpha \in J_{t}} \alpha^{\perp} \backslash\left\{n \in \mathbb{R}_{+}^{J_{t}} \cap \bigcup_{\alpha \in J_{t}} \alpha^{\perp}:\langle\nu, n\rangle \leqslant-\sup _{n^{1} \in \mathbb{R}_{+}^{\Delta \backslash J_{t}}}\left\langle\nu, n^{1}\right\rangle\right\}\right) \\
& =\partial \mathbb{R}_{+}^{J_{t}} \quad \text { ou } \partial \mathbb{R}_{+}^{J_{t}} \backslash\left\{n \in \partial \mathbb{R}_{+}^{J_{t}}:\langle\nu, n\rangle \leqslant 0\right\} .
\end{aligned}
$$

Donc $p\left(V \cap \bigcup_{\alpha \in J_{t}} \alpha^{\perp}\right)$ est un espace contractile sauf s'il est de la forme :

$$
(\diamond) \quad \partial \mathbb{R}_{+}^{J_{t}} \backslash\{0\}
$$

Dans ce cas, $p\left(V \cap \bigcup_{\alpha \in J_{t}} \alpha^{\perp}\right)$ est homéomorphe à $\mathbb{R}^{\left|J_{t}\right|-1} \backslash\{0\}$ et :

$$
\tilde{H}^{j-1}\left(V \cap \bigcup_{\alpha \in J_{t}} \alpha^{\perp}\right)= \begin{cases}0 & \text { si } j-1 \neq\left|J_{t}\right|-2 \\ \mathbb{R} & \text { si } j-1=\left|J_{t}\right|-2\end{cases}
$$

( $c f$. par exemple [32, chap. 4, théorème 6]).

On est dans cette situation $(\diamond)$ si et seulement si :

$$
\left\{n \in \partial \mathbb{R}_{+}^{J_{t}}:\langle\nu, n\rangle \leqslant 0\right\}=\{0\}
$$

et on vérifie que c'est équivalent à : $\nu \in Q_{t}$.

Finalement, pour tout $t \in W \backslash\{1\}$, tout $i \geqslant 0$ et tous $\lambda$, $\mu$, on retrouve que :

$$
H^{i-2 l(t)-1}\left(V(\lambda, t * \mu), V(\lambda, t * \mu) \cap \bigcup_{\alpha \in J_{t}} \alpha^{\perp}\right)=\mathbb{R}
$$

si $i=2 l(t)+\left|J_{t}\right|$ et $t * \mu \in \lambda+Q_{t}$ et que dans tous les autres cas :

$$
H^{i-2 l(t)-1}\left(V(\lambda, t * \mu), V(\lambda, t * \mu) \cap \bigcup_{\alpha \in J_{t}} \alpha^{\perp}\right)=\{0\} .
$$

Remarques et exemples. -

1. Pour tout poids entier dominant $\mu$ et tout faisceau inversible $\mathcal{L}$ sur $\overline{G_{a d}}$, la multiplicité du $\tilde{G} \times \tilde{G}$-module

$$
\bigoplus_{i \geqslant 0} H^{i}\left(\overline{G_{a d}}, \mathcal{L}\right)
$$

selon $\operatorname{End}(L(\mu))$ est majorée par l'ordre de $W$. 
2. Quel que soit le poids entier dominant $\mu$, l'ensemble des degrés $i$ en lesquels $H^{i}\left(\overline{G_{a d}}, \mathcal{L}\right)$ a une multiplicité non nulle selon $\operatorname{End}(L(\mu))$, pour au moins un faisceau inversible $\mathcal{L}$ est exactement :

$$
\left\{2 l(t)+\left|J_{t}\right|: t \in W\right\} .
$$

On en déduit, par exemple, que si $i=1,2$, ou 4 , alors :

$$
H^{i}\left(\overline{G_{a d}}, \mathcal{L}_{\lambda}\right)=\{0\}
$$

pour tout groupe $G$ et tout caractère $\lambda$.

3. Soit $\left\{\omega_{\alpha}: \alpha \in \Delta\right\}$ la base des poids fondamentaux. Posons pour toute partie $J$ de $\Delta$ :

$$
\begin{aligned}
P_{J} & :=\left\{\sum_{\alpha \in \Delta} p_{\alpha} \omega_{\alpha}: \forall \alpha, p_{\alpha} \in \mathbb{Z} \text { et }\left[p_{\alpha}<-1 \Leftrightarrow \alpha \in J\right]\right\}, \\
Q_{J} & :=\left\{\sum_{\alpha \in \Delta} q_{\alpha} \alpha: \forall \alpha, q_{\alpha} \in \mathbb{Z} \text { et }\left[q_{\alpha}>0 \Leftrightarrow \alpha \in J\right]\right\} .
\end{aligned}
$$

Avec ces notations, si $\mu$ est dominant, la bijection $w \mapsto w * \mu$ de $W$ sur $W * \mu$ induit une bijection :

$$
\left\{t \in W: t * \mu \in \lambda+Q_{t}\right\} \stackrel{\sim}{\rightarrow} \bigcup_{J \subseteq \Delta}\left\{\nu \in W * \mu \cap\left(\lambda+Q_{J}\right) \cap P_{J}\right\} .
$$

Si $\nu$ est un poids entier tel que $\nu+\rho$ est régulier, alors on note $\nu^{+}$l'unique poids dominant de $W * \nu$. On déduit de la bijection ci-dessus que l'ensemble des poids entiers $\nu$ tels que $\operatorname{End}\left(L\left(\nu^{+}\right)\right)$apparaisse dans la décomposition de la représentation :

$$
\bigoplus_{i \geqslant 0} H^{i}\left(\overline{G_{a d}}, \mathcal{L}_{\lambda}\right)
$$

est exactement l'ensemble :

$$
\bigcup_{J \subseteq \Delta}\left(\lambda+Q_{J}\right) \cap P_{J}
$$

4. On obtient aussi que, pour tous les groupes adjoints $G$ de rang 2 , les multiplicités $m_{\lambda}^{i}(\mu)$ sont 0 ou 1.

En effet, soit $\Delta:=\{\alpha, \beta\} ;$ soient $w_{1}, w_{2} \in W$ vérifiant :

$$
\left\{\begin{array}{l}
w_{j} * \mu \in \lambda+Q_{w_{j}} \\
2 l\left(w_{j}\right)+\left|J_{w_{j}}\right|=i
\end{array}\right.
$$

pour $j=1,2$ et un $0<i<\operatorname{dim} G$ (si $i=0$ ou $\operatorname{dim} G$, on sait déjà que les multiplicités $m_{\lambda}^{i}(\mu)$ sont 0 ou 1$)$.

On a alors $J_{w_{j}}=\{\alpha\}$ ou $\{\beta\}$. Si $J_{w_{1}}=J_{w_{2}}$, alors $l\left(w_{1}\right)=l\left(w_{2}\right)$. On vérifie que cela entraîne : $w_{1}=w_{2}$.

Si $J_{w_{1}} \neq J_{w_{2}}$, alors on a :

$$
\lambda+Q_{\{\alpha\}} \cap P_{\{\alpha\}} \neq \emptyset \quad \text { et } \quad \lambda+Q_{\{\beta\}} \cap P_{\{\beta\}} \neq \emptyset .
$$

Mais cela est impossible pour chacun des systèmes $A_{1} \times A_{1}, A_{2}, B_{2}, G_{2}$. 
5. On déduit aussi de la formule du théorème 2.2 que pour tout $G$ et tout caractère $\lambda \in \tilde{\mathcal{X}}$, les représentations $H^{3}\left(\overline{G_{a d}}, \mathcal{L}_{\lambda}\right)$ sont sans multiplicité. En effet, on a :

$$
2 l(w)+\left|J_{w}\right|=3 \quad \Leftrightarrow \quad \exists \alpha \in \Delta, w=s_{\alpha} .
$$

De plus, si $\mu \in \tilde{\mathcal{X}}^{+}, \lambda \in \tilde{\mathcal{X}}$ et $\alpha, \beta \in \Delta$, alors :

(**) $s_{\alpha} * \mu \in \lambda+Q_{s_{\alpha}} \quad$ et $\quad s_{\beta} * \mu \in \lambda+Q_{s_{\beta}} \Rightarrow s_{\alpha} * \mu-s_{\beta} * \mu \in Q_{\alpha}-Q_{\beta}$.

Mais comme :

$$
s_{\alpha} * \mu-s_{\beta} * \mu=\underbrace{-\left(\left\langle\mu, \alpha^{\vee}\right\rangle+1\right)}_{<0} \alpha+\underbrace{\left(\left\langle\mu, \beta^{\vee}\right\rangle+1\right)}_{>0} \beta
$$

et puisque :

$$
Q_{\alpha}-Q_{\beta} \subseteq \mathbb{Z}_{>0} \alpha-\mathbb{Z}_{>0} \beta+\mathbb{Z}(\Delta \backslash\{\alpha, \beta\}),
$$

il faut que $\alpha=\beta$ pour que la condition $(* *)$ soit vérifiée.

6. Les multiplicités $m_{\lambda}^{i}(\mu)$ peuvent néanmoins être différentes de 0 ou 1 : si par exemple, $G=P S O(8, \mathbb{C})$ (c'est le type $\left.D_{4}\right)$, on peut montrer à l'aide de 3 . que pour $i=5$ :

$$
\forall \mu \in \tilde{\mathcal{X}}^{+}, \quad \exists \lambda \in \tilde{\mathcal{X}}, \quad m_{\lambda}^{5}(\mu)=3 .
$$

7. Contrairement au cas du théorème de Borel-Weil-Bott, il peut arriver que $m_{\lambda}^{i}(\mu)>0$ pour plus d'un degré $i$ ( $\lambda$ et $\mu$ étant fixés). Par exemple, lorsque $G$ est le groupe adjoint de type $F_{4}$, on obtient grâce au théorème 2.2 qu'il existe une infinité de caractères $\lambda \in \tilde{\mathcal{X}}$ tels que $m_{\lambda}^{10}(0)>0$ et $m_{\lambda}^{11}(0)>0$.

Maintenant, on va démontrer le théorème 2.1.

On aura besoin de g, l'algèbre de Lie de G. On notera aussi $U(\mathfrak{g})$ son algèbre enveloppante.

On procède en trois grandes étapes. On commencera dans la section qui suit par rappeler la définition des groupes de cohomologie à support et le complexe de Grothendieck-Cousin, ceuxlà apparaissant dans celui-ci. Dans le contexte du fibré en droites $\mathcal{L}_{h}$ sur la compactification $\mathbf{X}$, ce complexe a un double intérêt. Non seulement son homologie est exactement la cohomologie $H^{*}\left(\mathbf{X}, \mathcal{L}_{h}\right)$, mais ses termes, qui sont des groupes de cohomologie à support, sont aussi des représentations de $\mathfrak{g} \times \mathfrak{g}$ dont on peut décrire précisément les sous-quotients simples de dimension finie, avec leurs multiplicités. On va étudier ces groupes de cohomologie au cours de la deuxième étape. Pour cela, on ne perdra rien en se plaçant dans le cadre plus général des variétés régulières. Grâce à cette étude, on pourra en la dernière étape éliminer la plupart des termes du complexe de Grothendieck-Cousin. Pour ceux qui resteront, on se ramènera d'abord à la détermination de groupes de cohomologie à support dans des variétés qui sont « presque des espaces affines ». Enfin pour conclure, on réduira tout à un calcul dans le contexte torique.

\section{Cohomologie à support}

Avec la définition des groupes de cohomologie à support, on redonne un résultat d'annulation qu'on utilisera souvent. 


\subsection{Définition et théorème d'annulation}

Soient $X$ un espace topologique et $Z_{2} \subseteq Z_{1} \subseteq X$ deux fermés de $X$. Soit $\mathcal{F}$ un faiseau abélien sur $X$.

DÉFINITION 3. - On note $\Gamma_{Z_{1} / Z_{2}}(\mathcal{F})$ le quotient de groupes abéliens :

$$
\left\{\sigma \in \mathcal{F}(X):\left.\sigma\right|_{X \backslash Z_{1}}=0\right\} /\left\{\sigma \in \mathcal{F}(X):\left.\sigma\right|_{X \backslash Z_{2}}=0\right\} \text {. }
$$

On appelle alors « $i$-ème groupe de cohomologie de $\mathcal{F}$ à support dans $Z_{1} / Z_{2}$ », et on note $H_{Z_{1} / Z_{2}}^{i}(\mathcal{F})$, le $i$-ème groupe dérivé à droite du foncteur :

$$
\mathcal{F} \mapsto \Gamma_{Z_{1} / Z_{2}}(\mathcal{F})
$$

Si $Z$ est seulement localement fermé dans $X$, on définit : $H_{Z}^{i}(\mathcal{F}):=H_{\bar{Z}}^{i} / \bar{Z} \backslash Z(\mathcal{F})$.

Cette notion généralise celle de cohomologie « tout court », en effet : $H_{X}^{i}(\mathcal{F})=H^{i}(X, \mathcal{F})$. À l'opposé, pour tout $i: H_{\emptyset}^{i}(\mathcal{F})=H_{Z / Z}^{i}(\mathcal{F})=\{0\}$.

On définit également, pour tout $i$, le $i$-ème groupe de cohomologie locale $\mathcal{H}_{Z_{1} / Z_{2}}^{i}(\mathcal{F})$ : c'est le faisceau associé au préfaisceau :

$$
U \mapsto H_{Z_{1} \cap U / Z_{2} \cap U}^{i}\left(\left.\mathcal{F}\right|_{U}\right)
$$

Dans le contexte des variétés on a :

ThÉorème 3.1. - Soit $X$ une variété lisse et irréductible, $Z$ une sous-variété affine de $X$, lisse et irréductible. Alors, pour tout faisceau $\mathcal{F}$ cohérent et localement libre sur $X$, on a:

$$
\forall i \neq \operatorname{codim}(Z, X), \quad H_{Z}^{i}(\mathcal{F})=\{0\}
$$

Démonstration. - D'une part, puisque $Z$ est une sous-variété affine, d'après [25, th. 9.5 d)] (ou $c f$. [34, annexe A1]), pour tout $i$ :

$$
H_{Z}^{i}(\mathcal{F})=\Gamma\left(X, \mathcal{H}_{Z}^{i}(\mathcal{F})\right)
$$

D'autre part, comme le faisceau $\mathcal{F}$ est localement libre et comme $Z$ et $X$ sont lisses, on a :

$$
\forall i \neq \operatorname{codim}(Z, X), \quad \mathcal{H}_{Z}^{i}(\mathcal{F})=0
$$

(cf. [18, th. 3.8] et [17, exp. III, lem. III.2]).

Remarque. - La condition « sous-variété affine, lisse et irréductible » est par exemple vérifiée par les $B$-orbites de $X$, pour un groupe connexe et résoluble $B$ qui opère sur $X$.

Rappelons aussi que pour une $G$-variété $X$, pour une sous-variété quelconque $Z$ de $X$ et pour un faisceau $\tilde{G}$-linéarisé sur $X, \mathrm{G}$. Kempf a montré que les groupes de cohomologie $H_{Z}^{i}(\mathcal{F})$ sont naturellement des g-modules ( $c f$. $[25,11.1,11.3,11.6])$.

\subsection{Théorème de Grothendieck-Cousin}

Ce théorème met en lumière le rôle de la cohomologie à support. 
THÉORÈME $3.2\left[25\right.$, th. 8.7 (b)]. $-{ }^{4}$ Soit $X$ un espace topologique. Soit $X \supseteq Z_{0} \supseteq Z_{1} \supseteq$ $\cdots \supseteq Z_{n} \supseteq Z_{n+1}=\emptyset$ une filtration de $X$ par des sous-espaces fermés.

(a) Pour tout faisceau $\mathcal{F}$ abélien sur $X$, on a un complexe, le « complexe de GrothendieckCousin » :

$$
0 \rightarrow H_{Z_{0} / Z_{1}}^{0}(\mathcal{F}) \stackrel{d^{0}}{\rightarrow} H_{Z_{1} / Z_{2}}^{1}(\mathcal{F}) \stackrel{d^{1}}{\rightarrow} \cdots \stackrel{d^{n-1}}{\rightarrow} H_{Z_{n}}^{n}(\mathcal{F}) \rightarrow 0
$$

(b) Si de plus la condition suivante est vérifiée :

$$
\forall p, \forall q \neq 0, \quad H_{Z_{p} / Z_{p+1}}^{p+q}(\mathcal{F})=\{0\} \quad(\star),
$$

alors pour tout $i \geqslant 0, H_{Z_{0}}^{i}(X, \mathcal{F})$ est le $i$-ème groupe d'homologie du complexe :

$$
0 \rightarrow H_{Z_{0} / Z_{1}}^{0}(\mathcal{F}) \stackrel{d^{0}}{\rightarrow} H_{Z_{1} / Z_{2}}^{1}(\mathcal{F}) \stackrel{d^{1}}{\rightarrow} \cdots \stackrel{d^{n-1}}{\rightarrow} H_{Z_{n}}^{n}(\mathcal{F}) \stackrel{d^{n}}{\rightarrow} 0 .
$$

D'après le théorème 3.1 , la condition $(\star)$ est vérifiée quand les trois suivantes sont remplies :

- $X$ est une variété algébrique lisse et irréductible,

- $\mathcal{F}$ est un faisceau cohérent et localement libre sur $X$,

- pour tout $p \geqslant 0$, la sous-variété $Z_{p} \backslash Z_{p+1}$ est vide, ou est lisse et de codimension pure $p$ dans $X$.

Appliquons ce théorème au faisceau inversible $\mathcal{L}_{h}$ et à la compactification régulière $\mathbf{X}$. On a donc besoin d'une filtration de $\mathbf{X}$ par des fermés. Notons $Z_{p}$ la réunion des $B \times B^{-}$-orbites de $\mathbf{X}$ de codimension $\geqslant p$ ( $p$ est un entier positif). On considérera la filtration :

$$
\mathbf{X}=Z_{0} \supseteq Z_{1} \supseteq \cdots \supseteq \cdots
$$

Puisque la variété $\mathbf{X}$ n'a qu'un nombre fini de $B \times B^{-}$-orbites $(c f .[3, \S 2.1])$ et que l'adhérence d'une orbite est une union d'orbites de codimension plus grande, pour tout $p \geqslant 0$, les $Z_{p}$ sont des fermés.

On va analyser le complexe de Grothendieck-Cousin qui apparaît :

$$
0 \rightarrow H_{Z_{0} / Z_{1}}^{0}\left(\mathcal{L}_{h}\right) \rightarrow H_{Z_{1} / Z_{2}}^{1}\left(\mathcal{L}_{h}\right) \rightarrow \cdots
$$

C'est un complexe de $\mathfrak{g} \times \mathfrak{g}$-modules dont le $p$-ième terme est :

$$
H_{Z_{p} / Z_{p+1}}^{p}\left(\mathcal{L}_{h}\right)=H_{Z_{p}-Z_{p+1}}^{p}\left(\mathcal{L}_{h}\right)=\bigoplus_{\Omega} H_{\Omega}^{p}\left(\mathcal{L}_{h}\right),
$$

cette somme se faisant sur les $B \times B^{-}$-orbites $\Omega$ de $\mathbf{X}$ de codimension $p$. De plus, comme le faisceau $\mathcal{L}_{h}$ est $\tilde{G} \times \tilde{G}$-linéarisé sur $\mathbf{X}$, pour toutes les $B \times B^{-}$orbites $\Omega$ de $\mathbf{X}$, l'action de l'algèbre de Lie de $B \times B^{-}$s'intègre en une action (rationnelle) du groupe $\tilde{B} \times \widetilde{B^{-}}$. On dit que les groupes $H_{\Omega}^{p}\left(\mathcal{L}_{h}\right)$ sont des $\mathfrak{g} \times \mathfrak{g}-\tilde{B} \times \widetilde{B^{-}}$-modules ( $c f$. [25, pp. 373, 374, 384]). Pour les étudier, on va en donner des filtrations. C'est l'objet de la partie qui suit.

\section{Filtration des groupes de cohomologie à support}

Dans toute cette partie, $X$ sera une variété complète et régulière (pour le groupe $G$ ).

\footnotetext{
${ }^{4}$ Dans [25], ce théorème est démontré dans le cas où $Z_{0}=X$; la généralisation ne pose aucun problème $(c f$. [11, lem. 1.2] ou [34, annexe A2]). 
Soit $L$ un fibré en droites $G$-linéarisé sur $X$. On va filtrer les groupes de cohomologie de $L$ à support dans une $B$-orbite $\Omega$ de $X$. Notre but est d'obtenir, comme gradués associés des g-modules très particuliers : des groupes de cohomologie de fibrés en droites sur un espace homogène $G / H$ et à support dans une $B$-orbite $B H / H$, avec un sous-groupe $H$ de $G$ contenant $T$. Pour ces groupes de cohomologie, le support $B H / H$ est un espace affine et on peut calculer leur caractère comme $T$-modules. De plus, lorsque $G / H$ est une variété projective (c'est-à-dire une variété de drapeaux), ces modules sont des représentations familières de $\mathfrak{g}$ : des modules de Verma généralisés.

En fait, on ne s'attaque pas directement aux groupes de cohomologie à support dans les $B$-orbites. On étudiera d'abord la cohomologie à support dans les cellules de BialynickiBirula (dont on rappelle la définition ci-dessous) : pour obtenir les filtrations voulues, c'est plus commode. Ensuite, on passera au cas où le support est une $B$-orbite. Enfin, on verra qu' on aboutit au cas évoqué ci-dessus, avec $G / H$ projective, lorsque tous les $T$-points fixes de $X$ sont dans une $G$-orbite fermée (comme on l'a vu, cette dernière condition est satisfaite par la compactification régulière $\mathbf{X})$.

\subsection{Les cellules et les orbites des variétés régulières}

Dans les variétés régulières, les cellules de Bialynicki-Birula jouent un rôle remarquable; elles permettent notamment de paramétrer les $B$-orbites et on les utilisera aussi pour les filtrations de groupes de cohomologie à support.

L'ensemble des points fixes de $T$ dans $X$ est fini (car d'une part, $X$ n'a qu'un nombre fini de $G$-orbites et d'autre part, le groupe de Weyl de $G$ opère transitivement sur l'ensemble des points fixes de chaque $G$-orbite). Notons le $X^{T}$. Il existe un sous-groupe à un paramètre $\zeta$ (de $T$ ), dominant (c'est-à-dire tel que pour tout $\alpha \in \Delta,\langle\alpha, \zeta\rangle \geqslant 0$ ) et dont les points fixes, dans $X$, sont aussi fixés par $T$ (cf. par exemple [5, lemme 2.3]).

Fixons pour toute cette partie un tel $\zeta$.

Soit $x \in X^{T}$. L'espace tangent à $X$ en $x, T_{x} X$, est un $T$-module. La cellule de BialynickiBirula

$$
X(x):=\left\{y \in X: \lim _{a \rightarrow 0} \zeta(a) \cdot y=x\right\}
$$

est une sous-variété de $X$, isomorphe à $\left(T_{x} X\right)_{+}$, le sous- $T$-module de $T_{x} X$ engendré par les vecteurs propres de poids «positifs» (i.e. ceux dont le crochet avec $\zeta$ est positif) (cf. [5]). On notera $\left(T_{x} X\right)_{-}$le sous- $T$-module de $T_{x} X$ engendré par les vecteurs propres de poids «négatifs ». Si $Z$ est une sous-variété fermée et $G$-invariante de $X$, on notera $Z(x):=X(x) \cap Z$ la cellule de Bialynicki-Birula de $Z$ associée à $x$ et à $\zeta$. Ces sous-variétés $X(x)$ (et $Z(x)$ ) sont $B$-invariantes car, pour tout $b \in B$, la limite

$$
\lim _{a \rightarrow 0} \zeta(a) b \zeta\left(a^{-1}\right)
$$

existe et appartient à $T$. Désormais, on appellera simplement cellules les cellules de BialynickiBirula associées aux points de $X^{T}$ et à $\zeta$.

On peut retrouver les $B$-orbites de $X$ grâce aux cellules. En effet, d'après $[10, \S 2.1$, p. 219 et prop. du §2.3], d'une part l'intersection d'une cellule et d'une $G$-orbite de $X$ est soit vide soit une $B$-orbite; d'autre part, on obtient ainsi toutes les $B$-orbites de $X$.

\subsection{Filtration de la cohomologie à support dans les cellules}

Soit $\mathcal{D}$ l'ensemble des diviseurs limitrophes de $X$ (ce sont les composantes irréductibles de $X \backslash X_{G}^{0}$, cf. la définition 1). Soit $x \in X^{T}$. Pour étudier les groupes de cohomologie à support 
dans les cellules, notre méthode consiste à filtrer selon l'ordre d'annulation le long des diviseurs limitrophes. Et, pour se ramener à de la cohomologie sur $G \cdot x$ et à support dans $B \cdot x$, on utilisera notamment que : $X(x) \cap \overline{G \cdot x}=B \cdot x$.

Soit $Z$ une sous-variété fermée, $G$-invariante et irréductible, de $\overline{G \cdot X(x)}$ qui contient $x$. On notera $z(x)$ la codimension de la cellule $Z(x)$ dans $X$ et $b(x)$ celle de l'orbite $B \cdot x$ dans l'orbite $G \cdot x$. D'après le théorème 3.1 , pour tout faisceau $\mathcal{M}$ localement libre et de rang fini sur $X$, on $\mathrm{a}: H_{Z(x)}^{i}(\mathcal{M})=\{0\}$ si $i \neq z(x)$. Le théorème suivant concerne le groupe de cohomologie à support $H_{Z(x)}^{z(x)}(\mathcal{M})$.

ThÉORÈME 4.1. - Soit $\mathcal{M}$ un faisceau cohérent, localement libre et $\tilde{G}$-linéarisé sur X. Pour tous $m \geqslant-1, n \geqslant 0$, on note $\mathcal{M}_{n}^{m}$ le faisceau $\bigoplus_{E} \mathcal{M}(E)$ où $E$ décrit l'ensemble des diviseurs de la forme:

$$
\sum_{\substack{D \in \mathcal{D} \\ Z \subseteq D}}\left(m_{D}+1\right) D-\sum_{\substack{D \in \mathcal{D} \\ x \in D, Z \nsubseteq D}} n_{D} D
$$

avec des entiers $m_{D}, n_{D} \geqslant 0$ vérifiant $: \sum_{D} m_{D}=m$ et $\sum_{D} n_{D}=n$.

Alors, avec ces notations, on a une filtration de g-modules:

$$
H_{Z(x)}^{z(x)}(\mathcal{M})=\bigcup_{m \geqslant-1, n \geqslant 0} F_{n}^{m},
$$

décroissante selon l'indice $n$, telle que, pour tous $m, n \geqslant 0$, on ait :

$$
F_{n}^{-1}=\{0\}, \quad \bigcap_{n \geqslant 0} F_{n}^{m}=F_{0}^{m-1}
$$

et dont les quotients successifs sont :

$$
F_{n}^{m} / F_{n+1}^{m}=H_{B \cdot x}^{b(x)}\left(\left.\mathcal{M}_{n}^{m}\right|_{G \cdot x}\right)
$$

Remargues. -

1. Dans la suite, on appliquera ce théorème à des faisceaux inversibles sur $X$.

2. Si on supprime l'hypothèse « $\tilde{G}$-linéarisé », on perd seulement le caractère g-équivariant de la filtration.

3. On a noté pour tout diviseur $D$ de $X, \mathcal{M}(D)$ le faisceau $\mathcal{M} \underset{D_{X}}{\otimes} \mathfrak{D}_{X}(D)$.

Démonstration. - Pour tout schéma $X^{\prime}$, le signe ${ }^{\vee}$ symbolisera le dual des $\mathfrak{D}_{X^{\prime}}$-modules. On emploiera aussi les notations suivantes : pour tous fermés $Z_{1} \subseteq Z_{2}$ de $X$,

- $\mathcal{I}_{Z_{1} / Z_{2}}$ le faisceau d'idéaux de définition de $Z_{1}$ dans $Z_{2}$ (et parfois, pour abréger : $\left.\mathcal{I}_{Z_{1}}:=\mathcal{I}_{Z_{1} / X}\right)$

- $\mathcal{N}_{Z_{1} / Z_{2}}:=\left(\mathcal{I}_{Z_{1} / Z_{2}} / \mathcal{I}_{Z_{1} / Z_{2}}^{2}\right)^{\vee}$ le faisceau normal de $Z_{1}$ par rapport à $Z_{2}$ (cf. [21, déf. du $\S 8.19])$;

- $\omega_{Z_{1} / Z_{2}}:=\bigwedge^{z_{2}-z_{1}} \mathcal{N}_{Z_{1} / Z_{2}}$ le faisceau canonique de $Z_{1}$ par rapport à $Z_{2}$, lorsque $Z_{1}$ et $Z_{2}$ sont lisses et irréductibles, de dimensions respectives $z_{1}$ et $z_{2}$ (cf. [20, déf. b), p. 141]).

On notera enfin $S^{p}$ la puissance symétrique $p$-ième.

Remarquons pour commencer que, d'après [3, th. du $\S 1.4$, ii)], $\overline{G . Z(x)}=Z$ et que, d'après [7, début du §2.4], on a des décompositions en faisceaux inversibles : 


$$
\begin{aligned}
& \left.\mathcal{N}_{Z / X}\right|_{\overline{G \cdot x}}=\left.\bigoplus_{D \in \mathcal{D}, D \supseteq Z} \mathfrak{D}_{X}(D)\right|_{\overline{G \cdot x}}, \\
& \left.\mathcal{N}_{G \cdot x / Z}\right|_{\overline{G \cdot x}}=\left.\bigoplus_{\substack{D \in \mathcal{D} \\
D \ni x, D \supseteq Z}} \mathfrak{D}_{X}(D)\right|_{\overline{G \cdot x}}, \\
& \left.\omega_{Z / X}\right|_{\overline{G \cdot x}}=\left.\mathfrak{D}_{X}\left(\sum_{D \in \mathcal{D}, D \supseteq Z} D\right)\right|_{\overline{G \cdot x}} .
\end{aligned}
$$

1. Cas où $Z=X$. On a alors : $\left.\mathcal{M}_{n}^{0}\right|_{\overline{G \cdot x}}=\mathcal{M} \otimes S^{n} \mathcal{N}_{\bar{G} \cdot x} / X$. On va filtrer selon l'ordre d'annulation le long de $\overline{G \cdot x}$ : on remarque, effectivement, que : $\mathcal{M}=\bigcup_{n \geqslant 0} \mathcal{M} \otimes \mathcal{I}_{\bar{G} \cdot x}$ d'où :

$$
H_{Z(x)}^{z(x)}(\mathcal{M})=\lim _{\vec{n} \geqslant 0} H_{Z(x)}^{z(x)}\left(\mathcal{M} \underset{\mathcal{D}_{X}}{\otimes} \mathcal{I} \frac{n}{G \cdot x}\right) .
$$

On va voir que cette limite directe est en fait une réunion (croissante) et on va en déterminer les quotients successifs. Pour tout $n \geqslant 0$, on a une suite exacte courte :

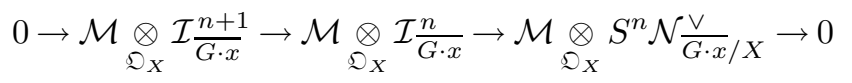

d'où l'on dérive une suite exacte :

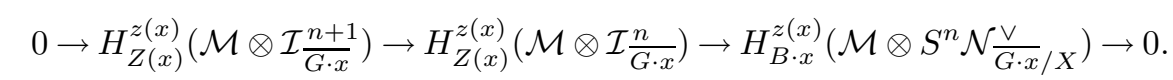

En effet, comme $Z(x)$ est une sous-variété affine et lisse de $X$, d'une part, on déduit du théorème 3.1 que pour $i \neq z(x), H_{Z(x)}^{i}\left(\mathcal{M} \otimes \mathcal{I}_{\bar{G} \cdot x}\right)=\{0\}$ et, d'autre part, comme $Z(x)$ et $\overline{G \cdot x}$ se coupent proprement dans $Z=X$ ( $c f$. [3, ii) du th. du $\S 1.4]$ ), avec $Z(x) \cap \overline{G \cdot x}=B \cdot x$, et $\mathcal{N}_{\overline{G \cdot x} / X}$ est localement libre sur $\overline{G \cdot x}$, on a de même :

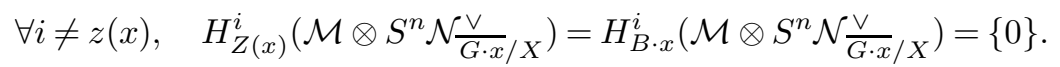

Posons alors : $F_{n}^{0}:=H_{Z(x)}^{z(x)}\left(\mathcal{M} \otimes \mathcal{I}_{\bar{G} \cdot x}^{n}\right)$ pour tout $n \geqslant 0$. On a bien :

$$
\bigcup_{n \geqslant 0} F_{n}^{0}=H_{Z(x)}^{z(x)}(\mathcal{M})
$$

et grâce à $(*)$ :

$$
F_{n}^{0} / F_{n+1}^{0}=H_{B \cdot x}^{z(x)}\left(\left.\mathcal{M}_{n}^{0}\right|_{\overline{G \cdot x}}\right)
$$

pour tout $n$.

Pour terminer, vérifions que $\bigcap_{n \geqslant 0} F_{n}^{0}=\{0\}$. Pour cela, on montre que la suite des caractères des $\tilde{T}$-modules $F_{n}^{0}$ tend vers 0 . On ne calcule pas exactement ces caractères mais on les majore; et c'est suffisant. On utilise les notations suivantes :

- si $\lambda \in \tilde{\mathcal{X}}$ (le réseau des caractères de $\tilde{T}), e^{\lambda}$ sera le caractère du $\tilde{T}$-module $\mathbb{C}_{\lambda}$, la droite $\mathbb{C}$ où $\tilde{T}$ agit via $\lambda$;

- si $M$ est un $\tilde{T}$-module, on notera $[M]$ son caractère, c'est-à-dire la somme formelle :

$$
\sum_{\lambda \in \tilde{\mathcal{X}}} \operatorname{dim} M_{\lambda} \cdot e^{\lambda}
$$


où, pour chaque $\lambda \in \tilde{\mathcal{X}}, M_{\lambda}$ est l'espace propre associé (ce sont les notations de [15, $\S \S 7.5 .1,7.5 .2])$.

Comme $Z(x)$ est une cellule de Bialynicki-Birula de $\overline{G \cdot x}$ et comme le faisceau $\mathcal{M}$ est cohérent, on a, d'après [34, th. II.3.2], l'inégalité suivante de caractères :

$$
\begin{aligned}
{\left[F_{n}^{0}\right] } & =\left[H_{Z(x)}^{z(x)}\left(\mathcal{M} \otimes \mathcal{I}_{\overline{G \cdot x}}^{n}\right)\right] \\
& \leqslant\left[\left(S\left(T_{x} X\right)_{+}\right)^{*}\right] \cdot\left[S\left(T_{x} X\right)_{-}\right] \cdot\left[\left.\omega_{Z(x) / \overline{G \cdot x}}\right|_{x}\right] \cdot\left[\left.\mathcal{M}\right|_{x}\right] \cdot\left[\left.\mathcal{I}_{\overline{G \cdot x}}\right|_{x}\right]
\end{aligned}
$$

pour chaque $n \geqslant 0$ (la lettre $S$ désigne la somme directe des puissances symétriques $p$-ièmes, sur tous les entiers naturels $p$ ).

Or, on a aussi l'inégalité :

$$
\left[\left.\mathcal{I}_{\overline{G \cdot x}}\right|_{x}\right] \leqslant\left[\left.\mathcal{I}_{\overline{G \cdot x}}\right|_{x}\right]^{n} .
$$

De plus, les poids du $\tilde{T}$-module $\left.\mathcal{I}_{\overline{G \cdot x}}\right|_{x}$ sont a fortiori des poids du $\tilde{T}$-module

$$
\Gamma\left(Z(x),\left.\mathcal{I}_{\overline{G \cdot x}}\right|_{Z(x)}\right)
$$

qui est un sous- $\tilde{T}$-module strict de $\mathcal{O}(Z(x))=\left(S\left(T_{x} X\right)_{+}\right)^{*}$ (car l'intersection $\overline{G \cdot x} \cap Z(x)$, contenant $x$, est non vide). Donc les poids $\lambda_{1}, \ldots, \lambda_{r}$ du $\tilde{T}$-module $\left.\mathcal{I}_{\overline{G \cdot x}}\right|_{x}$ vérifient tous : $\left\langle\lambda_{i}, \zeta\right\rangle<0$.

Il en résulte que la suite des caractères

$$
\left[\left.\mathcal{I} \overline{G \cdot x}\right|_{x}\right]^{n}=\left[e^{\lambda_{1}}+\cdots+e^{\lambda_{r}}\right]^{n}
$$

et, en conséquence, celle des caractères $\left[F_{n}^{0}\right]$ tendent vers 0 .

Cela termine la première étape.

2. On se ramène au cas de l'étape précédente. D'après [7, prop. 2.5], la variété $Z$ (comme toute adhérence de $G$-orbite) est encore régulière. Notons $z$ sa codimension dans $X$.

Puisque $Z(x) \subseteq Z$, grâce à [18, th. 2.8] et à [25, lem. 8.5.d)], on a :

$$
H_{Z(x)}^{z(x)}(\mathcal{M})=\lim _{m \geqslant 0} H_{Z(x)}^{z(x)-z}\left(\underline{\operatorname{Ext}}_{\mathfrak{D}_{X}}^{z}\left(\mathfrak{D}_{X} / \mathcal{I}_{Z}^{m}, \mathcal{M}\right)\right) .
$$

Cette limite directe est encore une réunion croissante. En effet, on tire de la suite exacte courte :

$$
0 \rightarrow S^{m} \mathcal{N}_{Z / X}^{\vee} \rightarrow \mathfrak{D}_{X} / \mathcal{I}_{Z}^{m+1} \rightarrow \mathfrak{D}_{X} / \mathcal{I}_{Z}^{m} \rightarrow 0
$$

une autre suite exacte de faisceaux :

$$
0 \rightarrow \underline{\operatorname{Ext}}_{\mathfrak{O}_{X}}^{z}\left(\mathfrak{D}_{X} / \mathcal{I}_{Z}^{m}, \mathcal{M}\right) \rightarrow \underline{\operatorname{Ext}}_{\mathfrak{O}_{X}}^{z}\left(\mathfrak{D}_{X} / \mathcal{I}_{Z}^{m+1}, \mathcal{M}\right) \rightarrow \underline{\operatorname{Ext}}_{\mathfrak{O}_{X}}^{z}\left(S^{m} \mathcal{N}_{Z / X}^{\vee}, \mathcal{M}\right) \rightarrow 0
$$

Or, puisque le faisceau $S^{m} \mathcal{N}_{Z / X}^{\vee}$ est localement libre sur $Z$, on a, d'après [20, prop. III.7.2] :

$$
\underline{\operatorname{Ext}}_{\mathfrak{O}_{X}}^{z}\left(S^{m} \mathcal{N}_{Z / X}^{\vee}, \mathcal{M}\right)=\omega_{Z / X} \otimes S^{m} \mathcal{N}_{Z / X} \otimes \mathcal{M}
$$

qui est un faisceau localement libre sur $Z$.

Donc, en posant $F^{m}:=H_{Z(x)}^{z(x)-z}\left(\underline{\operatorname{Ext}}_{\mathfrak{D}_{X}}^{z}\left(\mathfrak{D}_{X} / \mathcal{I}_{Z}^{m}, \mathcal{M}\right)\right)$, on obtient une filtration croissante $H_{Z(x)}^{z(x)}(\mathcal{M})=\bigcup_{m \geqslant 0} F^{m}$ de quotients succesifs :

$$
F^{m+1} / F^{m}=H_{Z(x)}^{z(x)-z}\left(\omega_{Z / X} \otimes S^{m} \mathcal{N}_{Z / X} \otimes \mathcal{M}\right) .
$$


On peut alors appliquer la première étape à la variété régulière $Z$ et au faisceau localement libre : $\omega_{Z / X} \otimes S^{m} \mathcal{N}_{Z / X} \otimes \mathcal{M}$. On trouve pour tout $m \geqslant-1$, une filtration décroissante $F^{m+1} / F^{m}=\bigcup_{n \geqslant 0} \bar{F}_{n}^{m}$. Puisque $\left.\mathcal{M}_{n}^{m}\right|_{\overline{G \cdot x}}=\omega_{Z / X} \otimes S^{m} \mathcal{N}_{Z / X} \otimes \mathcal{M} \otimes S^{n} \mathcal{N}_{\overline{G \cdot x} / Z}^{\vee}$, on a :

$$
\forall m, n, \quad \bar{F}_{n}^{m} / \bar{F}_{n+1}^{m}=H_{B \cdot x}^{b(x)}\left(\left.\mathcal{M}_{n}^{m}\right|_{G \cdot x}\right)
$$

On conclut la démonstration en prenant pour $F_{n}^{m}$ le relevé de $\bar{F}_{n}^{m}$ dans $F^{m+1}(m \geqslant-1)$.

Soit $\Omega$ une $B$-orbite de $X$, et $b$ sa codimension. On va maintenant exprimer les groupes de cohomologie à support dans $\Omega$ à l'aide de groupes de cohomologie à support dans une certaine cellule. Pour cela, on va utiliser que l'orbite $\Omega$ est l'intersection de la $G$-orbite $G . \Omega$ et d'une cellule $X(x)$. Ici, le point $x$ est la $\operatorname{limite} \lim _{a \rightarrow 0} \zeta(a)$.y pour un $y$ quelconque de $\Omega$ (cette limite est indépendante du $y$ choisi).

Notons $Z_{\Omega}$ l'adhérence de la $G$-orbite $G . \Omega$ et $D$ la somme des diviseurs limitrophes contenant $x$ mais non $\Omega$. Dans le théorème précédent, on a étudié les groupes $H_{Z_{\Omega}(x)}^{b}(\mathcal{M})$ pour un faisceau localement libre $\mathcal{M}$ sur $X$. Ils apparaissent dans la filtration de la proposition suivante :

Proposition 4.2. - Soit $\mathcal{M}$ un faisceau cohérent, localement libre et $\tilde{G}$-linéarisé sur X. Alors, on a une filtration croissante de g-modules:

$$
H_{\Omega}^{b}(\mathcal{M})=\bigcup_{k \geqslant 0} H_{Z_{\Omega}(x)}^{b}(\mathcal{M}(k D)) .
$$

Démonstration. - Puisque $G . \Omega=Z_{\Omega} \backslash D$, on a : $G . \Omega=Z_{\Omega}(x) \cap(X \backslash D)$. Or si on note $j: X \backslash D \hookrightarrow X$ l'inclusion canonique, on a :

$$
\lim _{k \geqslant 0} \mathcal{M}(k D)=\left.j_{*} \mathcal{M}\right|_{X \backslash D}
$$

Or, $j$ est un morphisme affine (il suffit de le vérifier pour chaque diviseur limitrophe, qui est lisse); d'où :

$$
H_{\Omega}^{b}(\mathcal{M})=H_{j^{-1}\left(Z_{\Omega}(x)\right)}^{b}\left(\left.\mathcal{M}\right|_{X \backslash D}\right)=H_{Z_{\Omega}(x)}^{b}\left(\left.j_{*} \mathcal{M}\right|_{X \backslash D}\right)=\lim _{\vec{k} \geqslant 0} H_{Z_{\Omega}(x)}^{b}(\mathcal{M}(k D)) .
$$

En outre, les morphismes :

$$
H_{Z_{\Omega}(x)}^{b}(\mathcal{M}(k D)) \rightarrow H_{Z_{\Omega}(x)}^{b}(\mathcal{M}((k+1) D))
$$

sont injectifs pour tout $k \geqslant 0$ (car chaque composante irréductible de $D$ coupe proprement $Z_{\Omega}(x)$ dans $Z_{\Omega}$ ).

Par conséquent, on a la filtration voulue.

\subsection{Suites de composition}

Soit $\mathcal{L}$ un faisceau $\tilde{G}$-linéarisé sur $X$. On suppose encore que $\Omega$ est une $B$-orbite de codimension $b$ dans $X$. En général, les g-modules $H_{\Omega}^{b}(\mathcal{L})$ ne sont pas de type fini. Néanmoins, ils admettent parfois une décomposition naturelle en somme directe de $\mathfrak{g}$-modules de longueur finie. C'est ce que nous allons maintenant étudier. 
Soit $Z(\mathfrak{g})^{\prime}$ l'ensemble des caractères du centre $Z(\mathfrak{g})$ de $U(\mathfrak{g})$. Rappelons $(c f .[15, \S 7.8 .15])$ que si $M$ est un $\mathfrak{g}$-module et si $\chi \in Z(\mathfrak{g})^{\prime}$, on peut définir, par récurrence, une suite croissante de sous-g-modules de $M$ en posant :

$$
M_{\chi}^{0}:=\{0\}, \quad \forall n>0, \quad M_{\chi}^{n}:=\left\{m \in M: \forall \mathbf{z} \in Z(\mathfrak{g}), \mathbf{z} \cdot m-\chi(\mathbf{z}) m \in M_{\chi}^{n-1}\right\} .
$$

On appelle la réunion croissante $M_{\chi}:=\bigcup_{n \geqslant 0} M_{\chi}^{n}$ l'espace propre généralisé de poids $\chi$ de $M$. Remarquons que les $M_{\chi}$ sont en somme directe lorsque $\chi$ décrit $Z(\mathfrak{g})^{\prime}$.

On reprend ici les notations du paragraphe 1.2.2 sur $\tilde{G}, \tilde{B}$, etc. On supposera dans cette partie que $\mathcal{L}$ est un faisceau $\tilde{G}$-linéarisé.

Pour chaque caractère central $\chi$ considérons l'espace propre généralisé $\left(H_{\Omega}^{b}(\mathcal{L})\right)_{\chi}$.

Suivant $[25$, pp. $373,374,384]$, les groupes $H_{\Omega}^{b}(\mathcal{L})$ sont des $\mathfrak{g}-\tilde{B}$-modules; c'est-à-dire que l'action de l'algèbre de Lie de $B$ (par restriction de celle de $G$ ) « s'intègre » en une action rationnelle de $\tilde{B}$. D'après $[15, \S 7.8 .15]$ ( $c f$. aussi $[34$, annexe $\mathrm{C} 1])$, il s'ensuit :

LEMME 4.3.-Comme g-modules :

$$
H_{\Omega}^{b}(\mathcal{L})=\bigoplus_{\chi \in Z(\mathfrak{g})^{\prime}}\left(H_{\Omega}^{b}(\mathcal{L})\right)_{\chi}
$$

On va montrer (proposition 4.6) que les espaces propres généralisés $\left(H_{\Omega}^{b}(\mathcal{L})\right)_{\chi}$ sont des g-modules de longueur finie lorsque le point $x$ fixé par $T$ et associé à la $B$-orbite $\Omega$ (c'est-à-dire l'unique $x$ tel que $\Omega=G . \Omega \cap X(x)$ ) appartient à une $G$-orbite fermée de $X$. Ce genre d'orbites $\Omega$ présente un avantage : dans les filtrations des groupes $H_{\Omega}^{b}(\mathcal{L})$ obtenues à la section précédente, ce sont des groupes de cohomologie de faisceaux sur l'orbite $G \cdot x$ qui interviennent comme quotients successifs, et cette orbite est une variété de drapeaux. Ces groupes de cohomologie sont, dans ce cas, des g-modules qui ont un caractère central connu, c'est ce que l'on rappelle, entre autres, dans la section qui suit. Par conséquent, en fixant un caractère central $\chi$, on obtiendra, grâce au théorème et à la proposition 4.1 et 4.2, une filtration explicite de l'espace propre généralisé correspondant : $\left(H_{\Omega}^{b}(\mathcal{L})\right)_{\chi}$. Cette filtration se trouvera être une suite de composition finie ( $c f$. la proposition 4.6).

Mais d'abord rappelons quelques $\mathfrak{g}-\tilde{B}$-modules particuliers :

\subsubsection{Les modules de Verma généralisés}

Soit $Q$ un sous-groupe parabolique de $G$ contenant $B^{-}$. Si $L \supseteq T$ est le sous-groupe de Levi correspondant, de racines positives $\Phi_{L}^{+}$, on notera

$$
W^{Q}:=\left\{w \in W: \forall \alpha \in \Phi_{L}^{+}, w(\alpha)>0\right\} .
$$

Ainsi, les $B$-orbites de la variété de drapeaux $G / Q$ sont les :

$$
B w Q / Q: w \in W^{Q}
$$

Remarque. - Pour tout $w \in W^{Q}, l(w)$ est la codimension de $B w Q / Q$ dans $G / Q$.

D'un autre côté, tout caractère $\lambda$ de $\tilde{Q}$ détermine un unique faisceau $\mathcal{L}$, inversible et $\tilde{G}$-linéarisé sur la variété $G / Q$ (c'est celui pour lequel $\tilde{Q}$ opère via $\lambda$ dans la fibre $\left.\mathcal{L}\right|_{Q / Q}$ ). Nous le noterons $\mathcal{L}_{G / Q}(\lambda)$. Le lemme suivant décrit les groupes de cohomologie à support $H_{B w Q / Q}^{l(w)}\left(\mathcal{L}_{G / Q}(\lambda)\right)$ comme g-modules. 
Pour l'énoncer, notons, pour tout caractère $\lambda \in \tilde{\mathcal{X}}, \chi_{\lambda}$ le caractère central avec lequel le centre $Z(\mathfrak{g})$ opère dans tous les $\mathfrak{g}$-modules engendrés par un vecteur de plus haut poids $\lambda$ ( $c f$. [15, prop. 7.4.4]).

ThÉORÈME 4.4 [12, §3, prop. 3] et [6, prop. 3.5]. - Pour tout caractère $\lambda$ de $\tilde{Q}$, le g $-\tilde{B}$ module

$$
H_{B w Q / Q}^{l(w)}\left(\mathcal{L}_{G / Q}(\lambda)\right)
$$

- admet $\chi_{\lambda}$ comme caractère central;

- a une suite de Jordan-Hölder (finie);

- a un unique plus haut poids $w * \lambda$ et celui-ci est de multiplicité 1 .

DÉFInition 4. - On notera $M_{Q}^{w}(\lambda)$ le g-module $H_{B w Q / Q}^{l(w)}\left(\mathcal{L}_{G / Q}(\lambda)\right)$.

Remarque. - Il résulte du théorème 4.4 que le g-module $M_{Q}^{w}(\lambda)$ a un $\tilde{G}$-module comme sousquotient simple si et seulement si $w * \lambda$ est dominant. De plus dans ce cas, le seul sous-quotient simple, qui est un $\tilde{G}$-module, est $L(w * \lambda)$ et sa multiplicité est 1 .

En effet, les sous-quotients simples de $M_{Q}^{w}(\lambda)$ sont de la forme $L(\nu)$ avec $\nu \in W * \lambda$ à cause du caractère central $\chi_{\lambda}$. Ensuite, parmi ces $\mathfrak{g}$-modules simples, un seul est de dimension finie : c'est le module $L(\nu)$ tel que $\nu$ est dominant. Enfin, la multiplicité du poids $w * \lambda$ dans chaque g-module $L(\nu)$ est nulle si $\nu \neq w * \lambda$ et vaut 1 dans $L(w * \lambda)$.

Si $Q=B^{-}, M_{B^{-}}^{w}(\lambda)$ est le module de Verma $w$-tordu $M^{w}(\lambda)$ de plus haut poids $w * \lambda$ défini dans $[16, \S 2.2]$.

\subsubsection{Quand le support est une orbite de rang maximal}

La notion d'orbites de rang maximal est définie par exemple dans $[4, \S 3]$. En fait, ce sont exactement les $B$-orbites $\Omega$ de $X$ telles que la $G$-orbite $G \cdot x$ du point limite :

$$
x=\lim _{a \rightarrow 0} \zeta(a) \cdot y \quad(\forall y \in \Omega)
$$

est fermée dans $X$ (cf. $[4, \S 3$, th. 3]).

On suppose dans ce paragraphe que $\Omega$ est une B-orbite de rang maximal.

Précisons quelques notations supplémentaires avant d'énoncer et de démontrer notre résultat sur les suites de composition des $\mathfrak{g}$-modules $\left(H_{\Omega}^{b}(\mathcal{L})\right)_{\chi}(b$ est toujours la codimension de l'orbite $\Omega)$.

Soit $Q$ le sous-groupe parabolique de $G$ contenant $B^{-}$associé à $X$ : c'est le sous-groupe parabolique opposé à $P$, le stabilisateur de la $B$-orbite ouverte de $X$; de plus, toutes les $G$-orbites fermées de $X$ sont isomorphes à $G / Q(c f .[7, \S 2.2]$ et $[3, \S 1.4])$. Puisque le point $x:=\lim _{a \rightarrow 0} \zeta(a) . y$ (pour tout $y \in \Omega$ ) est dans une $G$-orbite fermée de $X$, il existe un unique $w \in W^{Q}$ tel que $w . x$ soit un point fixe de $Q$ (dans $X$ ). Notons $w_{\Omega}$ ce $w$ et $z_{\Omega}$ ce point $w_{\Omega} . x$. Les faisceaux inversibles sur $X$ sont déterminés par leur restriction aux orbites fermées de $X$ et donc par leurs fibres en les points fixes de $Q$ dans $X$. Pour tout faisceau inversible et $\tilde{G}$-linéarisé sur $X, \mathcal{L}$, soit $p_{\Omega}(\mathcal{L})$ le caractère avec lequel le groupe $\tilde{Q}$ opère dans la fibre $\left.\mathcal{L}\right|_{z_{\Omega}}$.

Avec ces notations, il est immédiat que :

LEMME 4.5. - D'une part :

$$
b(x):=\operatorname{codim}(B \cdot x, G \cdot x)=\operatorname{codim}\left(B w_{\Omega} Q / Q, G / Q\right)=l\left(w_{\Omega}\right)
$$


et d'autre part, pour tout faisceau $\mathcal{L}$ inversible et $\tilde{G}$-linéarisé sur $X$, on a l'isomorphisme de g-modules:

$$
H_{B \cdot x}^{b(x)}\left(G \cdot x,\left.\mathcal{L}\right|_{G \cdot x}\right) \simeq M_{Q}^{w_{\Omega}}\left(p_{\Omega}(\mathcal{L})\right) .
$$

Pour un diviseur $B$-invariant $D$ de $X$, on notera $p_{\Omega}(D)$ le caractère $p_{\Omega}\left(\mathfrak{D}_{X}(D)\right)$.

Gardons les notations précédentes. $\mathrm{Si} \chi$ est un caractère central, l'ensemble des caractères $\nu \in \tilde{\mathcal{X}}$ tels que $\chi_{\nu}=\chi$ est une classe de $\tilde{\mathcal{X}}$ modulo l'action $*$ de $W$ (cf. [15, prop. 7.4.7]; notons la $W * \chi$. Du théorème et de la proposition sur les filtrations 4.1 et 4.2 , on déduit la :

Proposition 4.6. - Soit encore $\mathcal{D}$ l'ensemble des diviseurs limitrophes de X. On considère les parties suivantes de l'ensemble des caractères de $Q$ :

$$
\begin{aligned}
& I_{\Omega}:=\left\{p_{\Omega}(D): D \in \mathcal{D}, D \nsupseteq \Omega\right\}, \\
& J_{\Omega}:=\left\{p_{\Omega}(D): D \in \mathcal{D}, D \supseteq \Omega\right\} .
\end{aligned}
$$

On notera encore b la codimension de la $B$-orbite $\Omega$. Alors, pour tout faisceau $\mathcal{L}$, inversible et $\tilde{G}$-linéarisé sur $X$ et pour tout caractère central $\chi \in Z(\mathfrak{g})^{\prime}$, le $\mathfrak{g}$-module $\left(H_{\Omega}^{b}(\mathcal{L})\right)_{\chi}$ admet une suite de composition finie :

$$
\left(H_{\Omega}^{b}(\mathcal{L})\right)_{\chi}=F_{0} \supset F_{1} \supset \cdots \supset F_{N} \supset\{0\}
$$

dont les quotients successifs sont à permutation près les :

$$
M_{Q}^{w_{\Omega}}(\lambda): \lambda \in\left(p_{\Omega}(\mathcal{L})+\mathbb{Z} I_{\Omega}+\mathbb{Z}_{>0} J_{\Omega}\right) \cap W * \chi
$$

Remarque. - En particulier, les g-modules $\left(H_{\Omega}^{b}(\mathcal{L})\right)_{\chi}$ sont de longueur finie.

Démonstration.- Grâce au théorème 4.1 et à la proposition 4.2 , et avec leurs notations, on obtient une filtration infinie de $\mathfrak{g}$-modules :

$$
H_{\Omega}^{b}(\mathcal{L})=\bigcup_{m \geqslant-1, n, k \geqslant 0} F_{n}^{m, k}
$$

dont les quotients successifs sont :

$$
H_{B \cdot x}^{b(x)}\left(\left.\mathcal{M}_{n}^{m}(k D)\right|_{G \cdot x}\right) \quad(m \geqslant-1, n, k \geqslant 0) .
$$

Lorsque $(m, n, k)$ varie dans $\mathbb{Z}_{\geqslant-1} \times \mathbb{Z}_{\geqslant 0} \times \mathbb{Z}_{\geqslant 0}$, à l'aide du lemme 4.5 , on obtient comme quotients successifs les g-modules : $M_{Q}^{w_{\Omega}}(\lambda)$ où $\lambda$ décrit l'ensemble $p_{\Omega}(\mathcal{L})+\mathbb{Z} I_{\Omega}+\mathbb{Z}_{>0} J_{\Omega}$. (Remarquons que si $z_{\Omega} \notin D$, alors $p_{\Omega}(D)=0$.)

Maintenant, prendre la $\chi$-ième composante $\left(H_{\Omega}^{b}(\mathcal{L})\right)_{\chi}$ revient à ne garder, parmi ces $\mathrm{g}$-modules que ceux qui sont de caractère central $\chi:$ c'est-à-dire tels que : $\chi_{\lambda}=\chi$, ou encore $\lambda \in W * \chi$.

Le fait que chaque module de Verma généralisé apparaît au plus une fois dans la suite de composition est une conséquence de l'indépendance $\mathbb{Z}$-linéaire des caractères $p_{\Omega}(D), D \in \mathcal{D}$ (cf. [3, prop. A1, iv)]). On obtient une suite de composition finie car chaque ensemble $W * \chi$ est fini.

\subsubsection{Application}

En conséquence de la dernière proposition, on trouve le résultat suivant sur la structure des $\mathfrak{g} \times \mathfrak{g}$-modules $H_{B w B^{-}}^{l(w)}\left(\mathfrak{D}_{G}\right)$ pour tout $w \in W:$ 
Proposition 4.7. - Soient $G$ un groupe réductif et connexe sur $\mathbb{C}$ (avec les notations de la p. 2) et $w$ un élément du groupe de Weyl. Alors $H_{B w B^{-}}^{i}\left(\mathfrak{D}_{G}\right)=\{0\}$, pour $i \neq l(w)$, et les espaces propres généralisés non nuls de $H_{B w B^{-}}^{l(w)}\left(\Im_{G}\right)$ sont associés aux caractères centraux $\chi_{\lambda,-\lambda}, \lambda \in \tilde{\mathcal{X}}$. Chaque tel espace propre $H_{\lambda}^{w}$ (associé à $\chi_{\lambda,-\lambda}$ ) a une suite de composition finie de $\mathfrak{g} \times \mathfrak{g}$-modules :

$$
H_{\lambda}^{w}=F_{0} \supset F_{1} \supset \cdots \supset F_{N} \supset\{0\}
$$

où, à permutation près, les $F_{i} / F_{i+1}$ sont les :

$$
M^{w}(\mu) \otimes M(-\mu)^{*}, \quad \mu \in W * \lambda .
$$

Remarque. - On a noté $M(-\lambda)^{*}$ le dual restreint de $M(-\lambda)$, le module de Verma de plus bas poids $\lambda$ ( $c f$. par exemple le paragraphe qui précède la proposition 6 de [12]).

Démonstration.-Choisissons une compactification régulière $\mathbf{X}$ de $G$ et appliquons la proposition 4.6 à la variété $\mathbf{X}$ (qui est $G \times G$-régulière) et à la $B \times B^{-}$-orbite $\Omega=B w B^{-}-$. Aucun diviseur limitrophe ne contient l'orbite $B w B^{-}$. Ainsi, suivant les notations de la proposition précédente :

$$
I_{\Omega}=\left\{p_{\Omega}(D): D \in \mathcal{D}\right\}, \quad J_{\Omega}=\emptyset .
$$

De plus, d'après [3, prop. A1, iv)], le réseau $\mathbb{Z} I_{\Omega}$ engendré par les caractères $p_{\Omega}(D), D \in \mathcal{D}$, est aussi le réseau engendré par les $T \times T$-vecteurs propres de l'espace des fonctions rationnelles $\operatorname{sur} \bar{T}$ (ou sur $T$ ). C'est donc : $\{(\lambda,-\lambda): \lambda \in \mathcal{X}\}$.

Enfin, dans le cas particulier de la compactification régulière $\mathbf{X}$ de $G$, le groupe parabolique $Q$ associé à $\mathbf{X}(c f$. p. 435$)$ est le sous-groupe $B^{-} \times B$ de $G \times G$. On a ainsi, pour tout caractère $\lambda \in \tilde{\mathcal{X}}$, les égalités suivantes de $\mathfrak{g} \times \mathfrak{g}$-modules :

$$
\begin{aligned}
M_{Q}^{w_{\Omega}}(\lambda,-\lambda) & =M_{B^{-} \times B}^{(w, 1)}(\lambda,-\lambda)=H_{B w B^{-} / B^{-} \times B^{-} B / B}^{l(w)}\left(\mathcal{L}_{G / B^{-} \times G / B}(\lambda,-\lambda)\right) \\
& =H_{B w B^{-} / B^{-}}^{l(w)}\left(\mathcal{L}_{G / B^{-}}(\lambda)\right) \otimes H^{0}\left(B^{-} B / B, \mathcal{L}_{G / B}(-\lambda)\right) \\
& =M^{w}(\lambda) \otimes M(-\lambda)^{*} .
\end{aligned}
$$

Dans les deux dernières parties, on notera $U$ et $U^{-}$les sous-groupes unipotents maximaux de $B$ et $B^{-}$.

\section{Fin de la démonstration du théorème principal}

D'après [3, prop. A1, iv)], dans les compactifications de groupes réductifs, toutes les $B \times B^{-}$orbites sont de rang maximal.

On peut donc appliquer la proposition 4.6 dans ce cas.

Après avoir rappelé une paramétrisation des $B \times B^{-}$-orbites de $\mathbf{X}(5.1)$, on va se servir de la section précédente pour éliminer une grande partie des termes du complexe de GrothendieckCousin ( $c f$. la section 3.2) qui ne contribuent pas dans le calcul des multiplicités des groupes de cohomologie $H^{i}\left(\mathbf{X}, \mathcal{L}_{h}\right)$ (lemme 5.3). On exprimera alors ces multiplicités à l'aide de groupes de cohomologie à support dans certaines $B \times B^{-}$-sous-variétés de $\mathbf{X}$ (théorème 5.4). Enfin, on pourra exprimer, à leur tour, ces groupes de cohomologie à support simplement en fonction de la restriction de $\mathcal{L}_{h}$ à la variété torique $\bar{T} \cap \mathbf{X}_{0}$, d'éventail $\mathcal{E}^{+}$(5.4), et arriver ainsi à la formule du théorème 2.1 . 


\subsection{Les orbites dans la compactification}

Pour paramétrer les $B \times B^{-}$-orbites de $\mathbf{X}$, on utilise les cellules et les $G \times G$-orbites.

On choisit une fois pour toutes un sous-groupe à un paramètre $\zeta$ de $T \times T$, dominant et régulier relativement à $B \times B^{-}$et tel que tous les points fixés par $\zeta$ le soient aussi par $T \times T$.

D'après [3, prop. A1], les points fixes de $T \times T$ sont dans les $G \times G$-orbites fermées. Ce sont donc les points $(w, t) . z_{\sigma}$ avec $(w, t) \in W \times W$ et $\sigma$ un cône maximal de $\mathcal{E}^{+}$(rappelons que $z_{\sigma}$ est le point-base associé au cône $\sigma$ et que lorsque $\sigma$ décrit l'ensemble des cônes maximaux de $\mathcal{E}^{+}, z_{\sigma}$ décrit l'ensemble des points de $\mathbf{X}$ fixés par $B^{-} \times B$ ). Les $B \times B^{-}$-orbites de $\mathbf{X}$ sont dès lors les intersections non vides parmi les :

$$
\mathcal{O}_{w, t, \sigma}:=\mathcal{O} \cap \mathbf{X}\left((w, t) . z_{\sigma}\right)
$$

où $\mathcal{O}$ est une $G \times G$-orbite de $\mathbf{X}$, et $\mathbf{X}\left((w, t) . z_{\sigma}\right)$ est la cellule de Bialynicki-Birula associée au point $(w, t) . z_{\sigma}$ et au sous-groupe à un paramètre $\zeta(c f$. la section 4.1).

Pour la compactification magnifique $\overline{G_{a d}}$, il n'y a qu'un seul cône maximal : $\mathcal{C}^{+}$. On notera $\mathbf{X}_{a d}(w, t)$ la cellule de Bialynicki-Birula associée à $(w, t) \cdot z_{\mathcal{C}^{+}}$et à $\zeta$.

On se servira plus loin de l'ouvert $\mathbf{X}_{0}$ formé des $x \in \mathbf{X}$ tels que l'orbite $B \times B^{-} \cdot x$ est ouverte dans $G \times G \cdot x$ ( si $\mathbf{X}=\overline{G_{a d}}$, c'est la cellule ouverte). D'après [3, prop. A1], $\bar{T} \cap \mathbf{X}_{0}$ est la sous-variété torique ouverte de $\bar{T}$ d'éventail $\mathcal{E}^{+}$.

Plus généralement, on définit les relevés des cellules de $\overline{G_{a d}}$ dans $\mathbf{X}$ : suivant [3, prop. A2], comme $\mathbf{X}$ est régulière, la surjection canonique $G \longrightarrow G_{a d}$ se prolonge en un morphisme $G \times G$-équivariant $\pi: \mathbf{X} \rightarrow \overline{G_{a d}}$. Pour tous $w, t \in W$, soient $\mathbf{X}(w, t):=\pi^{-1}\left(\mathbf{X}_{a d}(w, t)\right)$.

Posons aussi :

$$
S(w, t):=\left(\left(w^{-1}, t^{-1}\right) \cdot \mathbf{X}(w, t)\right) \cap \bar{T} \cap \mathbf{X}_{0}
$$

(si $w=t=1$, alors : $\mathbf{X}(1,1)=\mathbf{X}_{0}$ et $\left.S(1,1)=\bar{T} \cap \mathbf{X}_{0}\right)$.

Ces variétés vérifient :

LEMme $5.1[10, \S 1.2$ et 2.3]. - Soient $w, t \in W$. Alors :

- $\mathbf{X}(w, t)$ est une sous-variété fermée de $(w, t) . \mathbf{X}_{0}$;

- $S(w, t)$ est une sous-variété fermée de $\bar{T} \cap \mathbf{X}_{0}$, formée des $s \in \bar{T} \cap \mathbf{X}_{0}$ tels que la limite quand a tend vers 0 de:

$$
\left(w^{-1}, t^{-1}\right)(\zeta)(a) . s
$$

existe dans $\bar{T} \cap \mathbf{X}_{0}$;

- on a la décomposition en $B \times B^{-}$-orbites :

$$
\mathbf{X}(w, t)=\bigsqcup_{\substack{\mathcal{O} G \times G \text {-orbite } \\ \sigma \in \mathcal{E}^{+} \text {maximal }}} \mathcal{O}_{w, t, \sigma} ;
$$

- l'application :

$$
\begin{aligned}
w U \times t U^{-} \times \bar{T} \cap \mathbf{X}_{0} & \longrightarrow \mathbf{X}, \\
(a, b, s) & \longmapsto(a, b) . s
\end{aligned}
$$


induit des isomorphismes de variétés algébriques :

$$
\begin{array}{r}
w U \times t U^{-} \times \bar{T} \cap \mathbf{X}_{0} \stackrel{\simeq}{\rightrightarrows}(w, t) \mathbf{X}_{0}, \\
(w U \cap U w) \times\left(t U^{-} \cap U^{-} t\right) \times S(w, t) \stackrel{\simeq}{\rightrightarrows} \mathbf{X}(w, t) .
\end{array}
$$

\section{Remarques. -}

1. Le dernier point du lemme a pour conséquence que, pour tout faisceau inversible et $\tilde{G} \times \tilde{G}$ linéarisé sur $\mathbf{X}$, on a un isomorphisme de faisceaux $\tilde{T} \times \tilde{T}$-linéarisés :

$$
\mathcal{L}_{h}\left|\mathbf{x}_{0} \simeq \Im_{U} \otimes \mathfrak{S}_{U^{-}} \otimes \mathcal{L}_{h}\right|_{T \cap \mathbf{x}_{0}}
$$

2. Lorsque $w=t$, l'éventail de la variété torique $\bar{T} \cap \mathbf{X}_{0} \backslash S(t, t)$ (ouverte dans $\bar{T}$ ) a un support indépendant du sous-groupe à un paramètre $\zeta$ choisi pour définir les cellules de BialynickiBirula; c'est l'ensemble :

$$
\bigcup_{\alpha \in \Delta: t(\alpha)<0} \alpha^{\perp} \cap \mathcal{C}^{+} .
$$

Pour établir ce fait, on va utiliser le lemme suivant démontré en annexe :

LEMme 5.2. - Soient $N$ un réseau de $\mathbb{R}^{n}$ et $\mathcal{E}$ un éventail lisse de $N$ (c'est-à-dire que tous ses cônes sont engendrés par un début de base de $N$ ). Soit $\gamma$ un élément de $N$. Nous considérons $\gamma$ comme un sous-groupe à un paramètre du tore $N \underset{\mathbb{Z}}{\otimes} \mathbb{C}$. On note $X(\mathcal{E})$ la variété torique associée à $\mathcal{E}$.

Alors, pour chaque cône $\sigma$ de $\mathcal{E}$ et son point-base $z_{\sigma}$, sont équivalentes :

(a) la limite de $\gamma(a) . z_{\sigma}$ existe dans $X(\mathcal{E})$ quand a tend vers 0 ;

(b) il existe un point $n_{0}$ dans l'intérieur du cône $\sigma$ tel que :

$$
\forall \epsilon>0, \exists 0<\eta<\epsilon \quad n_{0}+\eta \gamma \in|\mathcal{E}|
$$

$(|\mathcal{E}|$ est le support de $\mathcal{E})$.

Le sous-groupe à un paramètre $\zeta$ de $T \times T$ qui a servi à définir des cellules de Bialynicki-Birula $B \times B^{-}$-invariantes de $\mathbf{X}$ est de la forme $\zeta=\left(\zeta^{+}, \zeta^{-}\right)$, avec des sous-groupes à un paramètre réguliers de $T, \zeta^{+}$et $\zeta^{-}$qui sont respectivement dominants et antidominants (relativement à $B$ ). De plus, avec cette notation, un point-base $z_{\sigma}$ de $\bar{T} \cap \mathbf{X}_{0}$ appartient à l'ouvert $\bar{T} \cap \mathbf{X}_{0} \backslash S(t, t)$ si et seulement si la limite

$$
\lim _{a \rightarrow 0}\left(t^{-1}\left(\zeta^{+}\right)-t^{-1}\left(\zeta^{-}\right)\right)(a) . z_{\sigma}
$$

n'appartient pas à $\bar{T} \cap \mathbf{X}_{0}$.

Mais alors, d'après le lemme 5.2, le support de l'éventail de la variété torique $\bar{T} \cap \mathbf{X}_{0} \backslash S(t, t)$ est l'ensemble :

$$
\left\{n \in \mathcal{C}^{+}: \exists \epsilon>0, \forall 0<\eta<\epsilon, n+\eta t^{-1}\left(\zeta^{+}-\zeta^{-}\right) \notin \mathcal{C}^{+}\right\}
$$

(rappelons que $\mathcal{C}^{+}$est l'adhérence de la chambre de Weyl).

Or :

$$
n+\eta t^{-1}\left(\zeta^{+}-\zeta^{-}\right) \notin \mathcal{C}^{+} \quad \Leftrightarrow \quad \exists \alpha \in \Delta,\left\langle\alpha, n+\eta t^{-1}\left(\zeta^{+}-\zeta^{-}\right)\right\rangle<0
$$




$$
\begin{aligned}
& \Leftrightarrow \quad \exists \alpha \in \Delta, \eta\left\langle t(\alpha), \zeta^{+}-\zeta^{-}\right\rangle<-\langle\alpha, n\rangle \\
& \Leftrightarrow \quad \exists \alpha \in \Delta,\left\langle t(\alpha), \zeta^{+}-\zeta^{-}\right\rangle<0 \text { et } \eta>-\frac{\langle\alpha, n\rangle}{\left\langle t(\alpha), \zeta^{+}-\zeta^{-}\right\rangle} .
\end{aligned}
$$

Le sous-groupe à un paramètre $\zeta^{+}-\zeta^{-}$étant dominant et régulier, le signe de la racine $t(\alpha)$ est celui du crochet $\left\langle t(\alpha), \zeta^{+}-\zeta^{-}\right\rangle$. En conséquence, le support de la variété torique $\bar{T} \cap \mathbf{X}_{0} \backslash S(t, t)$ est :

$$
\left\{n \in \mathcal{C}^{+}: \exists \alpha \in \Delta, t(\alpha)<0 \text { et }\langle\alpha, n\rangle=0\right\}=\bigcup_{\alpha \in \Delta: t(\alpha)<0} \alpha^{\perp} \cap \mathcal{C}^{+} .
$$

\subsection{Calcul des multiplicités des groupes de cohomologie à support dans les orbites}

Soit $\mathcal{O}_{w, t, \sigma}$ une $B \times B^{-}$-orbite de $\mathbf{X}$ de codimension $i$. On s'intéresse aux sous-quotients simples (comme $\mathfrak{g} \times \mathfrak{g}$-modules) de dimension finie de $H_{\mathcal{O}_{w, t, \sigma}}^{i}\left(\mathcal{L}_{h}\right)$ et à leur multiplicité. On déduit de la proposition 4.6 le :

Lemme 5.3. - Soit L un $\tilde{G} \times \tilde{G}$-module simple. Si la multiplicité

$$
\left[H_{\mathcal{O}_{w, t, \sigma}}^{i}\left(\mathcal{L}_{h}\right): L\right]
$$

n'est pas nulle, alors : $w=t$ et il existe un caractère $\mu \in \tilde{\mathcal{X}}^{+}$tel que $L=\operatorname{End}(L(\mu))$.

Démonstration. - Soit $\chi$ le caractère central du $\mathfrak{g} \times \mathfrak{g}$-module $L$.

D'après la proposition 4.6 , si le $\mathfrak{g} \times \mathfrak{g}$-module $\left(H_{\mathcal{O}_{w, t, \sigma}}^{i}\right)_{\chi}$ a une multiplicité non nulle selon $L$, alors il en est de même pour un $\mathfrak{g} \times \mathfrak{g}$-module de la forme :

$$
M^{w}\left(h_{\sigma}+\delta\right) \otimes M^{t}\left(-h_{\sigma}-\delta\right)
$$

où $h_{\sigma}$ est le poids de la droite $\left.\mathcal{L}_{h}\right|_{z_{\sigma}}$ et, pour un certain diviseur $G \times G$-invariant $D$ de $\mathbf{X}, \delta$ est celui de la droite $\left.\mathcal{O}_{X}(D)\right|_{z_{\sigma}}$. Il est nécessaire, aussi, que :

$$
(w, t) *\left(h_{\sigma}+\delta,-\left(h_{\sigma}+\delta\right)\right) \in W * \chi .
$$

Soit $\left(\lambda_{1}, \lambda_{2}\right)$ le plus haut poids de $L$ relativement à $B \times B^{-}$(c'est-à-dire que $\lambda_{1}$ et $-\lambda_{2}$ sont dominants). On a alors :

$$
w\left(h_{\sigma}+\delta+\rho\right) \in W\left(\lambda_{1}+\rho\right) \quad \text { et } \quad t\left(-h_{\sigma}-\delta-\rho\right) \in W\left(-\lambda_{2}-\rho\right)
$$

d'où : $W\left(\lambda_{1}+\rho\right)=W\left(\lambda_{2}+\rho\right)$ et : $\lambda_{1}=-\lambda_{2}$ car $\lambda_{1}+\rho$ ainsi que $-\left(\lambda_{2}+\rho\right)$ sont dominants et réguliers. En conséquence, $L=\operatorname{End}\left(L\left(\lambda_{1}\right)\right)$.

D'un autre côté, pour que le $\mathfrak{g} \times \mathfrak{g}$-module $M^{w}\left(h_{\sigma}+\delta\right) \otimes M^{t}\left(-h_{\sigma}-\delta\right)$ ait un $\tilde{G} \times \tilde{G}$-module parmi ses sous-quotients simples, il faut que $w\left(h_{\sigma}+\delta+\rho\right)$ et $t\left(h_{\sigma}+\delta+\rho\right)$ soient dominants réguliers ( $c f$. la remarque après le théorème 4.4$)$. Cela entraîne que :

$$
w\left(h_{\sigma}+\delta+\rho\right)=t\left(h_{\sigma}+\delta+\rho\right)=\lambda_{1}=\lambda_{2}
$$

et que : $w=t$.

Grâce au complexe de Grothendieck-Cousin, il résulte immédiatement de ce lemme que, pour tout $i \geqslant 0$, les représentations simples de $\tilde{G} \times \tilde{G}$ qui apparaissent dans la décomposition du 
$\tilde{G} \times \tilde{G}$-module $H^{i}\left(\mathbf{X}, \mathcal{L}_{h}\right)$ sont de la forme $\operatorname{End}(L(\mu))$ pour un certain $\mu \in \tilde{\mathcal{X}}^{+}$. Ce lemme montre aussi que, dans le complexe de Grothendieck-Cousin et en vue du calcul des multiplicités des modules $\operatorname{End}(L(\mu))$ dans les groupes de cohomologie $H^{i}\left(\mathbf{X}, \mathcal{L}_{h}\right)$, on peut se passer des termes de la forme $H_{\mathcal{O}_{w, t, \sigma}}^{i}\left(\mathcal{L}_{h}\right)$, avec $w \neq t$. On se ramène ainsi, dans la section suivante, à une étude des groupes de cohomologie à support dans les variétés $\mathbf{X}(t, t), t \in W$. Cette étude est plus facile que l'étude directe des groupes $H^{i}\left(\mathbf{X}, \mathcal{L}_{h}\right)$ car, grâce au lemme d'excision, on peut remplacer $\mathbf{X}$ par n'importe quel ouvert qui contient $\mathbf{X}(t, t)$.

\subsection{Simplification de l'expression des multiplicités des groupes de cohomologie}

Avec les notations de la section précédente (5.1), on a :

THÉORÈme 5.4. - Pour tout $i \geqslant 0$ et tout $\mu \in \tilde{\mathcal{X}}^{+}$:

$$
\left[H^{i}\left(\mathbf{X}, \mathcal{L}_{h}\right): \operatorname{End}(L(\mu))\right]=\sum_{t \in W}\left[H_{\mathbf{X}(t, t)}^{i}\left(\mathcal{L}_{h}\right): \operatorname{End}(L(\mu))\right]
$$

Démonstration. - Soit $L$ un $\tilde{G} \times \tilde{G}$-module simple.

Suivant le théorème 3.2 , pour tout $i \geqslant 0, H^{i}\left(\mathbf{X}, \mathcal{L}_{h}\right)$ est le $i$-ème groupe d'homologie du complexe de Grothendieck-Cousin :

$$
K^{*}: 0 \rightarrow K^{0} \stackrel{d^{0}}{\rightarrow} K^{1} \stackrel{d^{1}}{\rightarrow} \cdots \rightarrow K^{\operatorname{dim} \mathbf{X}} \rightarrow 0
$$

où pour chaque $p \geqslant 0, K^{p}$ est le groupe de cohomologie à support $H_{Z_{p} \backslash Z_{p+1}}^{p}\left(\mathcal{L}_{h}\right)$ qui se décompose en la somme directe :

$$
\bigoplus_{\mathcal{O}, w, t, \sigma} H_{\mathcal{O}_{w, t, \sigma}}^{p}\left(\mathcal{L}_{h}\right)
$$

indexée par les $B \times B^{-}$-orbites de $\mathbf{X}$ de codimension $p$.

De plus, les applications $d^{i}$ définissent des morphismes (de $\mathfrak{g} \times \mathfrak{g}$-modules) :

$$
d^{\Omega, \Omega^{\prime}}: H_{\Omega}^{i}\left(\mathcal{L}_{h}\right) \rightarrow H_{\Omega^{\prime}}^{i+1}\left(\mathcal{L}_{h}\right)
$$

pour toutes paires d'orbites $\Omega, \Omega^{\prime}$ de codimensions $i$ et $i+1$.

Dans le complexe $K^{*}$, on ne va garder que les termes de la forme $H_{\mathcal{O}_{t, t, \sigma}}^{i}\left(\mathcal{L}_{h}\right)$; d'après le lemme 5.3, ce sont les seuls qui puissent avoir une multiplicité non nulle selon $L$ et on va voir qu'ils suffisent pour calculer les multiplicités voulues.

Plus précisément, pour tout entier $i$, soient $\mathcal{U}_{i}$ la réunion des $B \times B^{-}$-orbites de codimension $i$ et de la forme $\mathcal{O}_{t, t, \sigma}$, pour un certain $t \in W$, et $\mathcal{W}_{i}$ la réunion des $B \times B^{-}$-orbites de codimension $i$ et de la forme $\mathcal{O}_{w, t, \sigma}$, pour de certains $w, t \in W$ avec $w \neq t$.

Pour chaque $i, \mathcal{U}_{i}$ (respectivement $W_{i}$ ) est un sous-espace à la fois ouvert et fermé dans $Z_{i} \backslash Z_{i+1}$; on a donc :

$$
K^{i}=H_{\mathcal{U}_{i}}^{i}\left(\mathcal{L}_{h}\right) \oplus H_{\mathcal{W}_{i}}^{i}\left(\mathcal{L}_{h}\right) .
$$

Relativement à cette décomposition, on notera $\kappa_{i}$ l'injection

$$
H_{\mathcal{U}_{i}}^{i}\left(\mathcal{L}_{h}\right) \hookrightarrow H_{Z_{i} / Z_{i+1}}^{i}\left(\mathcal{L}_{h}\right)
$$


et $s_{i}$ la surjection :

correspondantes.

$$
H_{Z_{i} / Z_{i+1}}^{i}\left(\mathcal{L}_{h}\right) \longrightarrow H_{\mathcal{U}_{i}}^{i}\left(\mathcal{L}_{h}\right)
$$

Par composition, on obtient alors des morphismes :

$$
e^{i}:=s_{i+1} \circ d^{i} \circ \kappa_{i}: H_{\mathcal{U}_{i}}^{i}\left(\mathcal{L}_{h}\right) \rightarrow H_{\mathcal{U}_{i+1}}^{i+1}\left(\mathcal{L}_{h}\right)
$$

En ce qui concerne les multiplicités, ces morphismes vérifient la relation suivante :

$$
\left[H^{i}\left(\mathbf{X}, \mathcal{L}_{h}\right): L\right]=\left[h^{i}\left(K^{*}\right): L\right]=\left[\operatorname{ker} d^{i}: L\right]-\left[\operatorname{im} d^{i}: L\right]=\left[\operatorname{ker} e^{i}: L\right]-\left[\operatorname{im} e^{i}: L\right] .
$$

En fait, les morphismes $e^{i}$ se définissent aussi de la façon suivante :

La réunion disjointe $\mathcal{U}_{i} \sqcup \mathcal{U}_{i+1}$ est un sous-espace localement fermé de $\mathbf{X}$ où $\mathcal{U}_{i}$ est ouvert et $\mathcal{U}_{i+1}$ est fermé. En effet, l'espace $\mathcal{U}_{i} \sqcup \mathcal{U}_{i+1}$ est un ouvert de l'espace topologique $\mathcal{U}_{i} \cup Z_{i+1} \backslash Z_{i+2}$ (car le complémentaire de l'un dans l'autre est une union d'orbites de codimension maximale : 1), l'espace $\mathcal{U}_{i} \cup Z_{i+1} \backslash Z_{i+2}$ est un fermé de $Z_{i} \backslash Z_{i+2}$ (car le complémentaire de l'un dans l'autre est une union d'orbites de codimension minimale : 0 ) et $Z_{i} \backslash Z_{i+2}$ est localement fermé dans $\mathbf{X}$.

Le morphisme $e^{i}$ apparait alors dans la suite exacte longue naturelle :

$$
\cdots \rightarrow H_{\mathcal{U}_{i} \sqcup \mathcal{U}_{i+1}}^{i}\left(\mathcal{L}_{h}\right) \rightarrow H_{\mathcal{U}_{i}}^{i}\left(\mathcal{L}_{h}\right) \stackrel{e^{i}}{\rightarrow} H_{\mathcal{U}_{i+1}}^{i+1}\left(\mathcal{L}_{h}\right) \rightarrow \cdots
$$

(cf. [18, prop. 1.9]).

Considérons maintenant la suite de mophismes :

$$
\cdots \rightarrow H_{\mathcal{U}_{i-1}}^{i-1}\left(\mathcal{L}_{h}\right) \stackrel{e^{i-1}}{\rightarrow} H_{\mathcal{U}_{i}}^{i}\left(\mathcal{L}_{h}\right) \stackrel{e^{i}}{\rightarrow} H_{\mathcal{U}_{i+1}}^{i+1}\left(\mathcal{L}_{h}\right) \stackrel{e^{i+1}}{\rightarrow} \cdots
$$

On va voir que cette suite est en fait une somme directe de certains complexes de Grothendieck-Cousin.

Chaque morphisme $e^{i}$ a pour composantes les morphismes $d^{\Omega, \Omega^{\prime}}$ où $\Omega$ et $\Omega^{\prime} \operatorname{sont} \operatorname{des} B \times B^{-}$orbites de la forme $\mathcal{O}_{t, t, \sigma}$ et de codimensions respectives $i$ et $i+1$. D'un autre côté, pour des raisons de support, si un morphisme $d^{\Omega, \Omega^{\prime}}$ est non nul, alors on a :

$$
\Omega^{\prime} \subseteq \bar{\Omega} \text { et } \operatorname{codim}\left(\Omega^{\prime}, \bar{\Omega}\right)=1 .
$$

Or, de la description des adhérences des $B \times B^{-}$-orbites de $\mathbf{X}$ de [3, th. du §2.1] et [31, prop. 2.4], on déduit le :

Lemme 5.5 [34, prop. V.3.6]. - Soient $\mathcal{O}, \mathcal{O}^{\prime}$ des $G \times G$-orbites de $\mathbf{X}, w, w^{\prime} \in W$ et $\sigma, \sigma^{\prime}$ des cônes maximaux de $\mathcal{E}^{+}$.

Alors, si :

$$
\mathcal{O}_{w^{\prime}, w^{\prime}, \sigma^{\prime}}^{\prime} \subseteq \overline{\mathcal{O}}_{w, w, \sigma} \quad \text { et } \quad \operatorname{codim}\left(\mathcal{O}_{w^{\prime}, w^{\prime}, \sigma^{\prime}}^{\prime}, \overline{\mathcal{O}_{w, w, \sigma}} l\right)=1
$$

on $a: w=w^{\prime}$.

Admettons ce lemme pour le moment.

On a, pour tout $i \geqslant 0$ :

$$
\mathcal{U}_{i}=\bigsqcup_{t, \mathcal{O}, \sigma} \mathcal{O}_{t, t, \sigma}=\bigsqcup_{t \in W} \bigcup_{t, \sigma} \mathcal{O}_{t, t, \sigma}
$$


les unions se faisant sur les $B \times B^{-}$-orbites de codimension $i$. Soit alors pour chaque $t \in W$ :

$$
\mathbf{X} \supseteq \mathbf{X}(t, t) \supseteq Z_{0}^{t} \supseteq Z_{1}^{t} \supseteq \cdots
$$

la filtration de $\mathbf{X}(t, t)$ par ses sous-espaces fermés définis par :

$$
\forall i \geqslant 0, \quad Z_{i}^{t}:=\bigsqcup_{\substack{\mathcal{O}, \sigma \\ \operatorname{dim} \mathcal{O}_{t, t, \sigma} \leqslant \operatorname{dim} \mathbf{x}-i}} \mathcal{O}_{t, t, \sigma}
$$

Avec ces notations :

$$
\mathcal{U}_{i}=\bigsqcup_{t \in W}\left(Z_{i}^{t} \backslash Z_{i+1}^{t}\right) \quad \text { et } \quad H_{\mathcal{U}_{i}}^{i}\left(\mathcal{L}_{h}\right)=\bigoplus_{t \in W} H_{Z_{i}^{t} \backslash Z_{i+1}^{t}}^{i}\left(\mathcal{L}_{h}\right)
$$

et il résulte du lemme 5.5 que :

$$
e^{i}\left(H_{Z_{i}^{t} \backslash Z_{i+1}^{t}}^{i}\left(\mathcal{L}_{h}\right)\right) \subseteq H_{Z_{i+1}^{t} \backslash Z_{i+2}^{t}}^{i+1}\left(\mathcal{L}_{h}\right)
$$

pour tous $t, i$.

En conséquence, si on note $e_{t}^{i}$ la restriction du morphisme $e^{i}$ à $H_{Z_{i}^{t} \backslash Z_{i+1}^{t}}^{i}\left(\mathcal{L}_{h}\right)$, on a pour chaque $t \in W$ une suite de morphismes :

$$
K^{*}(t): 0 \rightarrow H_{Z_{0}^{t} \backslash Z_{1}^{t}}^{0}\left(\mathcal{L}_{h}\right) \stackrel{e_{t}^{0}}{\rightarrow} H_{Z_{1}^{t} \backslash Z_{2}^{t}}^{1}\left(\mathcal{L}_{h}\right) \stackrel{e_{t}^{1}}{\rightarrow} \cdots
$$

et pour chaque $i$ l'égalité :

$$
\left[\operatorname{ker} e^{i}: L\right]-\left[\operatorname{im} e^{i}: L\right]=\sum_{t \in W}\left[\operatorname{ker} e_{t}^{i}: L\right]-\left[\operatorname{im} e_{t}^{i}: L\right] .
$$

Or, pour chaque $t \in W$, on reconnaît en la suite $K^{*}(t)$ un complexe : le complexe de Grothendieck-Cousin associé à $\mathcal{L}_{h}$ et à la filtration $\left(Z_{i}^{t}\right)_{i \geqslant 0}$. Dès lors, d'après le théorème 3.2, on a des isomorphismes de $\mathfrak{g} \times \mathfrak{g}$-modules :

$$
\forall i \geqslant 0, \forall t \in W, \quad h^{i}\left(K^{*}(t)\right) \simeq H_{\mathbf{X}(t, t)}^{i}\left(\mathcal{L}_{h}\right)
$$

En résumé, si $i \geqslant 0$, alors :

$$
\left[H^{i}\left(\mathbf{X}, \mathcal{L}_{h}\right): L\right]=\left[h^{i}\left(K^{*}\right): L\right]=\sum_{t \in W}\left[h^{i}\left(K^{*}(t)\right): L\right]=\sum_{t \in W}\left[H_{\mathbf{X}(t, t)}^{i}\left(\mathcal{L}_{h}\right): L\right] .
$$

Voici maintenant la démonstration du lemme 5.5 :

On va tirer profit d'une particularité des orbites de la forme $\mathcal{O}_{w, w, \sigma}$. A priori, l'intersection de l'adhérence d'une $B \times B^{-}$-orbite et de l'adhérence d'une $G \times G$-orbite a plus d'une composante irréductible. Mais nous allons voir que si la $B \times B^{-}$-orbite est de la forme $\mathcal{O}_{w, w, \sigma}$, alors cette intersection est irréductible.

Avant cela, précisons quelques notations : si $\mathcal{O}$ est une $G \times G$-orbite de $\mathbf{X}, z_{\mathcal{O}} \in \bar{T}$ est toujours le point-base de l'orbite $\mathcal{O}$. Soit $\theta$ un sous-groupe à un paramètre de $T$ tel que $\lim _{a \rightarrow 0} \theta(a)=z_{\mathcal{O}}$; on pose :

$$
L(\mathcal{O}):=\left\{g \in G: \forall a \in \mathbb{C}^{*}, \theta(a) g \theta(a)^{-1}=g\right\}
$$


c'est le sous-groupe de Levi, contenant $T$, du groupe parabolique :

$$
P(\mathcal{O}):=\left\{g \in G: \lim _{a \rightarrow 0} \theta(a) g \theta(a)^{-1} \text { existe dans } G\right\} ;
$$

tout cela est indépendant du $\theta$ choisi (cf. [3, prop. A1]). Enfin, on note $W_{L(\mathcal{O})}$ le groupe de Weyl de $L(\mathcal{O})$ et $W^{L(\mathcal{O})}:=\left\{w \in W: \forall v \in W_{L(\mathcal{O})}, l(w v)=l(w)+l(v)\right\} .^{5}$

Cela étant posé, si $\mathcal{O}_{w, w, \sigma}$ est une $B \times B^{-}$-orbite de $\mathbf{X}$ (avec $w \in W$ et $\sigma$ un cône maximal de $\mathcal{E}^{+}$, alors, d'après $[3, \S 1.1$ et $\S 2.1]$, c'est la $B \times B^{-}$-orbite du point $(w, w) . z_{\mathcal{O}}$; de plus, $w \in W^{L(\mathcal{O})}$.

Or, d'après [3, (ii) du théorème du §2.1], si $\mathcal{O}^{\prime}$ est une $G \times G$-orbite de $\overline{\mathcal{O}}$, alors l'intersection $\overline{\mathcal{O}^{\prime}} \cap \overline{B \times B^{-}(w, w) . z_{\mathcal{O}}}$ est propre dans $\overline{\mathcal{O}}$ et ses composantes irréductibles sont de la forme :

$$
\overline{B \times B^{-}(w v, w v) . z_{\mathcal{O}}} \quad \text { avec } v \in W_{L(\mathcal{O})} \text { tel que } l(w)=l(w v)+l(v) .
$$

Mais alors on a $: l(w v)=l(w)+l(v)\left(\operatorname{car} w \in W^{L(\mathcal{O})}\right.$ et $\left.v \in W_{L(\mathcal{O})}\right)$ et donc $: l(w)=$ $l(w)+2 l(v)$, d'où $: v=1$.

En particulier, $\overline{\mathcal{O}^{\prime}} \cap \overline{B \times B^{-}(w, w) . z_{\mathcal{O}}}=\overline{B \times B^{-}(w, w) z_{\mathcal{O}^{\prime}}}$ est irréductible.

Supposons maintenant que $\mathcal{O}_{w^{\prime}, w^{\prime}, \sigma^{\prime}}^{\prime} \subseteq \overline{\mathcal{O}_{w, w, \sigma}}$. On a alors : $\mathcal{O}^{\prime} \subseteq \overline{\mathcal{O}}=G \times G \overline{\mathcal{O}_{w, w, \sigma}}$. Soit $\mathcal{O}_{\sigma^{\prime}}$ la $G \times G$-orbite fermée correspondant à $\sigma^{\prime}$; on a :

$$
\begin{aligned}
& \mathcal{O}_{\sigma^{\prime}} \subseteq \overline{\mathcal{O}^{\prime}} \subseteq \overline{\mathcal{O}} \quad \text { et }: \\
& \overline{\mathcal{O}_{w^{\prime}, w^{\prime}, \sigma^{\prime}}^{\prime}} \cap \overline{\mathcal{O}_{\sigma^{\prime}} \subseteq \overline{\mathcal{O}_{w, w, \sigma}} \cap \mathcal{O}_{\sigma^{\prime}}} \\
& \Leftrightarrow \quad \overline{B \times B^{-}\left(w^{\prime}, w^{\prime}\right) \cdot z_{\sigma^{\prime}}} \subseteq \overline{B \times B^{-}(w, w) \cdot z_{\sigma^{\prime}}} \\
& \Leftrightarrow \overline{B w^{\prime} B^{-} / B^{-}} \times \overline{B^{-} w^{\prime} B / B} \subseteq \overline{B w B^{-} / B^{-}} \times \overline{B^{-} w B / B} \\
& \Leftrightarrow \quad w^{\prime} \geqslant w
\end{aligned}
$$

selon l'ordre de Bruhat.

Or, comme $\overline{\mathcal{O}_{w, w, \sigma}}$ et $\mathcal{O}_{\sigma}$ s'intersectent proprement dans $\overline{\mathcal{O}}$, on a :

$$
\operatorname{codim}\left(\mathcal{O}_{w, w, \sigma}, \mathbf{X}\right)=\operatorname{codim}\left(\overline{\mathcal{O}_{w, w, \sigma}} \cap \mathcal{O}_{\sigma}, \mathcal{O}_{\sigma}\right)+\operatorname{codim}(\mathcal{O}, \mathbf{X})=2 l(w)+\operatorname{codim}(\mathcal{O}, \mathbf{X})
$$

De même $: \operatorname{codim}\left(\mathcal{O}_{w, w, \sigma}, \mathbf{X}\right)=2 l\left(w^{\prime}\right)+\operatorname{codim}\left(\mathcal{O}^{\prime}, \mathbf{X}\right)$.

Par conséquent, si on suppose aussi que $\operatorname{codim}\left(\mathcal{O}_{w^{\prime}, w^{\prime}, \sigma^{\prime}}^{\prime}, \overline{\mathcal{O}_{w, w, \sigma}}\right)=1$, on a :

$$
2\left(l\left(w^{\prime}\right)-l(w)\right)+\operatorname{codim}\left(\mathcal{O}^{\prime}, \mathcal{O}\right)=1
$$

avec $l\left(w^{\prime}\right)-l(w) \geqslant 0$ et $\operatorname{codim}\left(\mathcal{O}^{\prime}, \mathcal{O}\right) \geqslant 0$. Cela n'est possible que si $l\left(w^{\prime}\right)=l(w)$ i.e. si $w^{\prime}=w$.

\subsection{Réduction au cas torique}

Pour conclure, il reste à déterminer les multiplicités

$$
\left[H_{\mathbf{X}(t, t)}^{i}\left(\mathcal{L}_{h}\right): \operatorname{End}(L(\mu))\right]
$$

\footnotetext{
${ }^{5} W_{L(\mathcal{O})}$ est le sous-groupe de $W$ engendré par les réflexions simples $s_{\alpha}(\alpha \in \Delta)$ telles que : $\langle\alpha, \theta\rangle=0$ et $W^{L(\mathcal{O})}$ est l'ensemble des $w \in W$ tels que $w(\alpha)>0$ pour tout $\alpha \in \Delta$ vérifiant $\langle\alpha, \theta\rangle=0$. 
lorsque $t \in W$.

On va se ramener au calcul du caractère d'un groupe de cohomologie à support d'un faisceau inversible sur une variété torique.

Grâce aux isomorphismes du lemme 5.1, on a un isomorphisme de $\tilde{T} \times \tilde{T}$-modules :

$$
H_{\mathbf{X}(t, t)}^{i}\left(\mathcal{L}_{h}\right) \simeq H_{t U \cap U t}^{l(t)}\left(\mathfrak{D}_{U t}\right) \otimes H_{t U^{-} \cap U^{-} t}^{l(t)}\left(\mathfrak{D}_{U^{-} t}\right) \otimes H_{S(t, t)}^{i-2 l(t)}\left(\left.\mathcal{L}_{h}\right|_{\bar{T} \cap \mathbf{X}_{0}}\right) .
$$

Et, en utilisant les isomorphimes de $\tilde{T}$-modules suivants ( $c f$. [25, lemme 12.8$]$ ou [28, lemme 3.16] pour les deux premiers) :

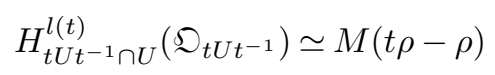

(le module de Verma de plus haut poids $t \rho-\rho$ )

$$
H_{t U^{-} t^{-1} \cap U^{-}}^{l(t)}\left(\mathfrak{D}_{t U^{-} t^{-1}}\right) \simeq M(-t \rho+\rho)
$$

(le module de Verma de plus bas poids $-t \rho+\rho$ ), et

$$
H_{S(t, t)}^{i-2 l(t)}\left(\left.\mathcal{L}_{h}\right|_{\bar{T} \cap \mathbf{X}_{0}}\right) \simeq \bigoplus_{\nu \in \tilde{\mathcal{X}}} m_{\nu} \mathbb{C}_{\nu}
$$

(pour certains entiers $m_{\nu}{ }^{6}$ ), on obtient un isomorphisme de $\tilde{T} \times \tilde{T}$-modules :

$$
H_{\mathbf{X}(t, t)}^{i}\left(\mathcal{L}_{h}\right) \simeq \bigoplus_{\nu \in \tilde{\mathcal{X}}} m_{\nu}(M(t(\nu+\rho)-\rho) \otimes M(-(t(\nu+\rho)-\rho)))
$$

Mais, comme les caractères des $\mathfrak{g} \times \mathfrak{g}$-modules simples $L$ (à plus hauts poids) sont $\mathbb{Z}$-linéairement indépendants, on trouve que, pour tout caractère $\mu \in \tilde{\mathcal{X}}^{+}$, la multiplicité selon $\operatorname{End}(L(\mu))$ de $H_{\mathbf{X}(t, t)}^{i}\left(\mathcal{L}_{h}\right)$ vérifie :

$$
\left[H_{\mathbf{X}(t, t)}^{i}\left(\mathcal{L}_{h}\right): \operatorname{End}(L(\mu))\right]=\sum_{\nu \in W * \mu} m_{\nu}[M(t(\nu+\rho)-\rho): L(\mu)]=m_{t^{-1}(\mu+\rho)-\rho}
$$

( $c f$. la remarque qui suit le théorème 4.4).

Dès lors, pour tout $i \geqslant 0$ et pour tout $\mu \in \tilde{\mathcal{X}}^{+}$, on a :

$$
\left[H^{i}\left(\mathbf{X}, \mathcal{L}_{h}\right): \operatorname{End}(L(\mu))\right]=m_{\mu}+\sum_{t \in W, t \neq 1} m_{t^{-1} * \mu} .
$$

Enfin, on rappelle ( $c f$. la remarque 2 qui suit le lemme 5.1) que les variétés toriques $\bar{T} \cap \mathbf{X}_{0}$ et $\bar{T} \cap \mathbf{X}_{0} \backslash S(t, t)$ ont pour éventails : $\mathcal{E}^{+}$avec $\left|\mathcal{E}^{+}\right|=\mathcal{C}^{+}$et un ensemble $\mathcal{E}_{t}^{+}$avec

$$
\left|\mathcal{E}_{t}^{+}\right|=\bigcup_{\alpha \in J_{t-1}} \alpha^{\perp} \cap \mathcal{C}^{+}
$$

La formule de [30, th. 2.6] ( $c f$. aussi [34, th. II.4.2]) permet alors d'exprimer les entiers $m_{t^{-1} * \mu}$ en fonction de $h$ et des sous-espaces topologiques $\mathcal{C}^{+}$et $\bigcup_{\alpha \in J_{t}} \alpha^{\perp} \cap \mathcal{C}^{+}$de $\mathcal{Y}_{\mathbb{R}}$.

\footnotetext{
$\overline{{ }^{6} \text { On considère } H_{S(t, t)}^{i}-2 l(t)}\left(\left.\mathcal{L}_{h}\right|_{\bar{T} \cap \mathbf{X}_{0}}\right)$ comme un $\tilde{T}$-module grâce à l'action de $\tilde{T} \times\{1\}$ sur $\bar{T} \cap \mathbf{X}_{0}$ et sur $S(t, t)$.
} 
Cela achève la démonstration du théorème 2.1 .

En fait, pour la cohomologie des fibrés en droites sur les variétés toriques, la méthode du complexe de Grothendieck-Cousin aboutit directement à une formule qui fait intervenir la cohomologie d'Ishida ( $c f$. [34, th. II.4.4]).

\section{Remerciement}

Je suis très reconnaissant à mon directeur de thèse, Michel Brion, de sa relecture attentive et patiente, et des améliorations et corrections qu'il m'a fait apporter aux premières versions. Un grand merci également à Syu Kato pour les bons échanges que nous avons eus.

\section{Annexe A. Un résultat sur les variétés toriques lisses}

On va démontrer le lemme 5.2. On va utiliser les notations introduites dans l'énoncé ainsi que les notations usuelles concernant la combinatoire des variétés toriques ( $c f$. par exemple [30]) : en particulier, la lettre $M$ désignera le réseau dual de $N$.

(a) $\Rightarrow$ (b) Si la limite existe dans $X(\mathcal{E})$, elle appartient à un ouvert affine de la forme $\Omega_{\tau}=\operatorname{Spec}\left(\mathbb{C}\left[M \cap \tau^{\vee}\right]\right)$ pour un certain cône $\tau$ de l'éventail $\mathcal{E}$.

Forcément, $\sigma$ est une face de $\tau$ et, puisque $\mathcal{E}$ est lisse, il existe une base $\left(e_{1}, \ldots, e_{n}\right)$ de $N$ telle que :

$$
\tau=\mathbb{R}_{+} e_{1}+\cdots+\mathbb{R}_{+} e_{t} \quad \text { et } \quad \sigma=\mathbb{R}_{+} e_{1}+\cdots+\mathbb{R}_{+} e_{s}
$$

avec des entiers $s \leqslant t \leqslant n$. Pour tout $a \in \mathbb{C}^{*}$, on peut identifier le point $\gamma(a) . z_{\sigma}$ de $\operatorname{Spec}\left(\mathbb{C}\left[M \cap \tau^{\vee}\right]\right)$ avec le morphisme additif :

$$
\begin{aligned}
M \cap \tau^{\vee} & \longrightarrow \mathbb{C}, \\
m & \longmapsto \begin{cases}a^{\langle m, \gamma\rangle} & \text { si } m \in M \cap \tau^{\vee} \cap \sigma^{\perp}, \\
0 & \text { sinon. }\end{cases}
\end{aligned}
$$

Pour que cela converge dans la variété $X(\mathcal{E})$, quand $a$ tend vers 0 , il est nécessaire et suffisant que :

$$
\forall m \in M \cap \tau^{\vee} \cap \sigma^{\perp}, \quad\langle m, \gamma\rangle \geqslant 0 .
$$

Or, si on note $\left(e_{1}^{*}, \ldots, e_{n}^{*}\right)$ la base duale de $\left(e_{1}, \ldots, e_{n}\right)$, on trouve que :

$$
\tau^{\vee} \cap \sigma^{\perp}=\mathbb{R}_{+} e_{s+1}^{*}+\cdots+\mathbb{R}_{+} e_{t}^{*}+\mathbb{R} e_{t+1}+\cdots+\mathbb{R} e_{n}^{*}
$$

La condition $(*)$ équivaut donc à :

$$
\gamma \in \mathbb{R} e_{1}+\cdots+\mathbb{R} e_{s}+\mathbb{R}_{+} e_{s+1}+\cdots+\mathbb{R}_{+} e_{t} .
$$

Écrivons $\gamma$ sous la forme $\gamma_{1} e_{1}+\cdots+\gamma_{t} e_{t}$ avec $\gamma_{1}, \ldots, \gamma_{s} \in \mathbb{R}$ et $\gamma_{s+1}, \ldots, \gamma_{t} \geqslant 0$. Soit $n_{0}:=e_{1}+\cdots+e_{s}$. C'est un élément de l'intérieur du cône $\sigma$ et, si $\eta>0$, on a :

$$
\begin{aligned}
n_{0}+\eta \gamma \in \tau & \Leftrightarrow \quad\left(1+\eta \gamma_{1}\right) e_{1}+\cdots+\left(1+\eta \gamma_{s}\right) e_{s}+\eta \gamma_{s+1} e_{s+1}+\cdots+\eta \gamma_{t} e_{t} \in \tau \\
& \Leftrightarrow \quad \forall 1 \leqslant i \leqslant s, \eta \gamma_{i} \geqslant-1 .
\end{aligned}
$$

Cela est toujours vérifié, quel que soit $\epsilon>0$, pour au moins un $\eta$ dans l'intervalle $] 0, \epsilon[$. A fortiori pour un tel $\eta, n_{0}+\eta \gamma$ appartient au support de $\mathcal{E}$. Ceci démontre le sens « $\Rightarrow »$. 
(a) $\Leftarrow$ (b) Soit $n_{0}$ un point de l'intérieur de $\sigma$ tel que la condition (b) du lemme soit satisfaite. Comme le support $|\mathcal{E}|$ est une réunion finie de cônes $\tau \in \mathcal{E}$, il existe un tel cône $\tau$ et une suite $\left(\eta_{k}\right)_{k \in \mathbb{N}}$ de réels strictement positifs tels que :

$$
\forall k \geqslant 0, \quad n_{0}+\eta_{k} \gamma \in \tau
$$

En faisant tendre $k$ vers l'infini, on s'aperçoit que $n_{0}$ appartient à $\tau$. Par conséquent, $\tau$ rencontre l'intérieur de $\sigma$; ce qui n'est possible que si $\sigma$ est une face de $\tau$.

Comme nous l'avons vu dans la démonstration du sens « $\Rightarrow »$, pour que la limite en 0 de $\gamma(a) . z_{\sigma}$ existe dans l'ouvert $\operatorname{Spec}\left(\mathbb{C}\left[M \cap \tau^{\vee}\right]\right)$ de $X(\mathcal{E})$, il suffit que :

$$
\forall m \in M \cap \tau^{\vee} \cap \sigma^{\perp}, \quad\langle m, \gamma\rangle \geqslant 0 .
$$

Or, si $m \in M \cap \tau^{\vee} \cap \sigma^{\perp}$, on a :

$$
\left\langle m, n_{0}+\eta_{0} \gamma\right\rangle=\left\langle m, n_{0}\right\rangle+\eta_{0}\langle m, \gamma\rangle=\eta_{0}\langle m, \gamma\rangle
$$

car $m \in \sigma^{\perp}$ et $n_{0} \in \sigma$. D'un autre côté $\left\langle m, n_{0}+\eta_{0} \gamma\right\rangle \geqslant 0$ car $m \in \tau^{\vee}$ et $n_{0}+\eta_{0} \gamma \in \tau$. Ainsi, puisque $\eta_{0}>0$, on a : $\langle m, \gamma\rangle \geqslant 0$. Cela achève la démonstration du lemme 5.2.

\section{RÉFÉRENCES}

[1] BRION M., Groupe de Picard et nombres caractéristiques des variétés sphériques, Duke Math. J. 58 (2) (1989) 397-424.

[2] BRIOn M., Une extension du théorème de Borel-Weil, Math. Ann. 286 (4) (1990) 655-660.

[3] BRION M., The behaviour at infinity of the Bruhat decomposition, Comment. Math. Helv. 73 (1998) 137-174.

[4] BRION M., On orbit closures of spherical subgroups in flag varieties, Comment. Math. Helv. (2001) 263-299.

[5] BiAlyniCKi-Birula A., Some theorems on actions of algebraic groups, Ann. Math. 98 (1973) 480497.

[6] Borho W., Brylinski J.-L., Differential operators in homogeneous spaces, I, Invent. Math. 69 (1982) 437-476.

[7] BIEN F., BRION M., Automorphisms and local rigidity of regular varieties, Compositio Math. 104 (1996) 1-26.

[8] Bifet E., On complete symmetric varieties, Adv. Math. 80 (1990) 225-249.

[9] Bifet E., De Concini C., Procesi C., Cohomology of regular embeddings, Adv. Math. 82 (1990) $1-34$.

[10] Brion M., Luna D., Sur la structure locale des variétés sphériques, Bull. Soc. Math. France 115 (1987) 211-226.

[11] BozICEVIC M., A geometric construction of a resolution of the fundamental series, Duke Math. J. 60 (3) (1990) 643-669.

[12] BRYLINSKI J.-L., Differential operators on the flag varieties, in: Young tableaux and Schur functors, in : Astérisque, vol. 87-88, 1981, pp. 43-60.

[13] De Concini C., Procesi C., Complete symmetric varieties, in : Invariant Theory, Proc. 1 st 1982 Sess. C.I.M.E., Montecatini/Italie, in : Lect. Notes Math., vol. 996, 1983, pp. 1-44.

[14] Demazure M., Sous-groupes algébriques de rang maximum du groupe de Cremona, Ann. Sci. Norm. Sup. (4) 3 (1970) 507-588.

[15] DiXmIER J., Algèbres enveloppantes, Cahiers scientifiques, Fasc. XXXVII, Gauthier-Villars, Paris, 1974. 
[16] Feigin B.L., Frenkel E.V., Affine Kac-Moody algebras and semi-infinite flag manifolds, Comm. Math. Phys. 128 (1990) 161-189.

[17] Grothendieck A., Cohomologie locale des faisceaux cohérents et théorèmes de Lefschetz locaux et globaux, Séminaire de géométrie algébrique, fasc. I, 1962.

[18] Grothendieck A., Local Cohomology, in : Lect Notes Math., vol. 41, Springer, Berlin, 1967.

[19] Godement R., Théorie des faisceaux, Hermann, Paris, 1958.

[20] Hartshorne R., Residues and Duality, in : Lect. Notes Math., vol. 20, Springer, Berlin, 1966.

[21] Hartshorne R., Algebraic Geometry, $8^{e}$ éd., Springer, 1997.

[22] IVERSEN B., A fixed point formula for action of tori on algebraic varieties, Invent. Math. 16 (1972) 229-236.

[23] IVERSEN B., The geometry of algebraic groups, Adv. Math. 20 (1976) 57-85.

[24] Kato S., A Borel-Weil-Bott type theorem for group completions, J. Algebra 259 (2003) 572-580.

[25] KempF G., The Grothendieck-Cousin complex of an induced representation, Adv. Math. 29 (1978) 310-396.

[26] Knop F., Kraft H., LunA D., Vust T., Local properties of algebraic group actions, in: Kraft H., Slodowy P., Springer T.A. (Eds.), Algebraic Transformation Groups and Invariant Theory, in: DMV Seminar, vol. 13, Birkhäuser, Basel, 1989.

[27] Knop F., Kraft H., Vust T., The Picard group of a $G$-variety, in : Kraft H., Slodowy P., Springer T.A. (Eds.), Algebraic Transformation Groups and Invariant Theory, in : DMV Seminar, vol. 13, Birkhäuser, Basel, 1989.

[28] KUMAR S., Bernstein-Gelfand-Gelfand resolution for arbitrary Kac-Moody algebras, Math. Ann. 286 (1990) 709-729.

[29] Luna D., Vust Th., Plongements d'espaces homogènes, Comment. Math. Helv. 58 (1983) 186-245.

[30] OdA T., Convex Bodies and Algebraic Geometry, Springer, Berlin, 1988.

[31] SPRINGER T.A., Intersection cohomology of $B \times B$-orbits in group compactifications, J. Algebra 258 (2002) 71-111.

[32] SpAnier E.H., Algebraic Topology, in: McGraw-Hill Series in Higher Mathematics, New York, vol. XIV, 1966.

[33] Tchoudjem A., Cohomologie des filbrés en droites sur la compactification magnifique d'un groupe semi-simple adjoint, C. R. Math. Acad. Sci. Paris 334 (6) (2002) 441-444.

[34] TCHOudJEM A., Représentations d'algèbres de Lie dans des groupes de cohomologie à support, Thèse de doctorat, Grenoble, 2002.

\footnotetext{
Alexis TCHOUdJEM

Institut Desargues,

Université de Lyon (I),

43, boulevard du Onze-Novembre 1918,

69622 Villeurbanne Cedex, France

Courriel: Alexis.Tchoudjem@igd.univ-lyon1.fr
} 